This is an electronic reprint of the original article. This reprint may differ from the original in pagination and typographic detail.

Author(s): Almosly, Wafa; Carlsson, B. G.; Suhonen, Jouni; Toivanen, Jussi; Ydrefors, Emanuel

Title: $\quad$ Theoretical estimates of supernova-neutrino cross sections for the stable even-even lead isotopes: Charged-current reactions

Year: $\quad 2016$

Version:

Please cite the original version:

Almosly, W., Carlsson, B. G., Suhonen, J., Toivanen, J., \& Ydrefors, E. (2016).

Theoretical estimates of supernova-neutrino cross sections for the stable even-even lead isotopes: Charged-current reactions. Physical Review C, 94(4), Article 044614. https://doi.org/10.1103/PhysRevC.94.044614

All material supplied via JYX is protected by copyright and other intellectual property rights, and duplication or sale of all or part of any of the repository collections is not permitted, except that material may be duplicated by you for your research use or educational purposes in electronic or print form. You must obtain permission for any other use. Electronic or print copies may not be offered, whether for sale or otherwise to anyone who is not an authorised user. 


\title{
Theoretical estimates of supernova-neutrino cross sections for the stable even-even lead isotopes: Charged-current reactions
}

\author{
W. Almosly, ${ }^{1}$ B. G. Carlsson, ${ }^{2}$ J. Suhonen, ${ }^{1}$ J. Toivanen, ${ }^{1}$ and E. Ydrefors ${ }^{1,3}$ \\ ${ }^{1}$ University of Jyvaskyla, Department of Physics, P.O. Box 35, FI-40014 Jyvaskyla, Finland \\ ${ }^{2}$ Division of Mathematical Physics, LTH, Lund University, P.O. Box 118, S-22100 Lund, Sweden \\ ${ }^{3}$ Instituto Tecnológico de Aeronaáutica, DCTA, 12228-900, São José dos Campos, São Paulo, Brazil \\ (Received 15 June 2016; revised manuscript received 22 August 2016; published 24 October 2016)
}

\begin{abstract}
A detailed study of the charged-current supernova electron neutrino and electron antineutrino scattering off the stable even-mass lead isotopes $A=204,206$, and 208 is reported in this work. The proton-neutron quasiparticle random-phase approximation (pnQRPA) is adopted to construct the nuclear final and initial states. Three different Skyrme interactions are tested for their isospin and spin-isospin properties and then applied to produce (anti)neutrino-nucleus scattering cross sections for (anti)neutrino energies below $80 \mathrm{MeV}$. Realistic estimates of the nuclear responses to supernova (anti)neutrinos are computed by folding the computed cross sections with a two-parameter Fermi-Dirac distribution of the electron (anti)neutrino energies. The computed cross sections are compared with earlier calculations and the analyses are extended to take into account the effects coming from the neutrino oscillations.
\end{abstract}

DOI: 10.1103/PhysRevC.94.044614

\section{INTRODUCTION}

The neutrino is a neutral particle introduced by Pauli in 1930 to restore the energy conservation in beta decay and given the name "neutrino" by Fermi in 1932. Since that time, the neutrino and its properties have attracted great interest in theoretical and experimental studies of particle and nuclear physics. Neutrino experiments provide evidence of neutrino oscillations; therefore, the neutrino can no longer be considered a massless particle. However, the absolute value of the neutrino mass is still an open question [1]. Further questions, such as the nature of neutrino, i.e., being a Dirac or a Majorana particle, and the mass hierarchy, still remain unanswered [1].

Neutrino-interaction cross sections are truly important ingredients in any neutrino experiment. Knowledge of these cross sections offers a valuable probe to investigate various questions in particle and astrophysics. Nuclear responses to supernova neutrinos, for example, are important to investigate supernova mechanisms $[2,3]$ and the nucleosynthesis of heavy elements [4,5]. Furthermore, the estimations of neutrinonucleus cross sections constitute a significant tool for detecting different neutrino flavors and exploring the structure of the weak interactions $[6,7]$.

Neutrinos interact only weakly with matter, and neutrinos from astrophysical sources, such as supernovae, can therefore be detected by Earth-bound detectors via charged-current (CC) and/or neutral-current (NC) neutrino-nucleus interactions. The CC experiments concentrate on detection of electron neutrinos $\left(v_{e}\right)$ and antineutrinos $\left(\bar{v}_{e}\right)$. Several neutrino detectors around the world are being established and planned for such a purpose; see, e.g., [3] for an overview on supernova-neutrino detectors. One example of such a detector is HALO (Helium and Lead Observatory) [8] running at SNOLAB, Canada, and designed for observation of galactic core-collapse supernovae by a lead-based neutrino detector. The HALO experiment is complementary to other neutrino-detection experiments in that it is dominated by $v_{e}$ events over the $\bar{v}_{e}$ events, since $v_{e}$ events are enhanced by the large neutron excess of the $\mathrm{Pb}$ nuclei and $\bar{v}_{e}$ events are suppreseed by Pauli blocking [9]. Hence, theoretical estimates of neutrino-nucleus responses for stable lead targets are essential for the interpretation of the results from HALO and similar detection experiments.

In addition to the above neutrino-nucleus reactions, studies of neutrinoless double- $\beta$ decay can provide significant information on the unknown neutrino properties such as the neutrino mass [10] and the Majorana nature of the neutrino [11]. Also, the estimation of charged-current neutrino-nucleus cross sections is important for probing the nuclear matrix elements of neutrinoless double- $\beta$ decay by exploiting the so-called neutrino beams [12].

The present work is a continuation of our efforts to provide realistic estimates of neutrino-nucleus interactions in various nuclei. In our previous works we have studied neutrino and antineutrino scatterings off molybdenum isotopes [13-16], cadmium isotopes [17-19], and ${ }^{136} \mathrm{Xe}$ [20]. Thus, the aim of the present work is to estimate and study in detail the charged-current supernova (anti)neutrino scattering off the stable even-even lead isotopes of mass numbers $A=204$, 206, and 208. We adopted the proton-neutron quasiparticle random-phase approximation (pnQRPA) to construct the nuclear states which are relevant for the (anti)neutrino-nucleus reactions. We perform self-consistent calculations within a valence space consisting of 15 major harmonic oscillator shells using the code HOSPHE [21]. Our computations are based on three different globally parametrized Skyrme interactions, namely SkM*, SkX, and SLy4 [22]. The (anti)neutrino-nucleus scattering calculations are based on the Donnelly-Walecka method for the treatment of semileptonic processes in nuclei [23]. The nuclear responses to electron (anti)neutrinos from a supernova are estimated subsequently by folding the cross sections with realistic energy profiles for the incoming supernova-(anti)neutrino flux. Concerning the lead nuclei, the present work is an extension of the works $[24,25]$ where only 
${ }^{208} \mathrm{~Pb}$ of the lead isotopes was discussed. We will then compare our present results with the ones of the aforementioned works for the isotope ${ }^{208} \mathrm{~Pb}$. In the end we extend our discussion to viable scenarios where the neutrino oscillations in dense supernova matter are taken into account. Earlier studies of this kind suggest that megaton-scale terrestrial experiments on the supernova electron antineutrino flux could be used to determine the mass hierarchy of the neutrino, as suggested in [19] and visible also in the results of [20].

The rest of this paper is organized as follows. At first, in Sec. II we briefly outline the main theoretical formalism of the adopted nuclear-structure framework and the charged-current (anti)neutrino-nucleus cross sections. Then, in Sec. III, we present and discuss our obtained results. Finally, in Sec IV, we summarize our main conclusions.

\section{THEORY}

\section{A. Nuclear-structure calculations}

In the nuclear-structure calculations the ground state is obtained using the Hartree-Fock-Bogolubov (HFB) approach. The nuclear effective interaction is approximated with the Skyrme interaction. This interaction consists of the lowestorder terms in an expansion in momentum space and should be a reasonable approximation when the relative velocities of the interacting nucleons are not too large. In addition it contains a density-dependent part that approximates missing effects such as three-body interactions and correlation corrections that go beyond the many-body treatment.

Three different parametrizations of the Skyrme force are investigated. The SkX parametrization has been tuned with special emphasis on describing the level structure in a set of spherical nuclei [26]. The $\mathrm{SkM}^{*}$ parametrization is tuned to describe collective excitations and deformation properties $[27,28]$ and the SLy4 interaction has been constructed with specific attention to the neutron equation of state [29].

Coulomb and center-of-mass-motion corrections are treated as specified by the different Skyrme parametrizations. For the Coulomb interaction, the direct part of the Coulomb potential is taken into account while Coulomb exchange is treated in the Slater approximation. In order to correct the kinetic energy for center-of-mass motion, a one-body correction is generally adopted.

In addition, we employ a gaussian type pairing interaction in a separable form [30]. The pairing interaction is parametrized with two different strengths $\left(G_{0}, G_{1}\right)$ for the $T=0$ and $T=1$ channels [31]. For the range of the interaction we adopt the value $a=0.660 \mathrm{fm}$ [30]. The contribution from $T=0$ pairing in the HFB ground states is neglected. This part of the pairing can lead to pn pairing condensates in $Z=N$ nuclei if the interaction strength is taken to be strong enough.

The excitations are calculated using the same interaction as used for the ground state and are obtained using the pnQRPA method in combination with iterative Arnoldi diagonalization [31].

Considering the spectra of a set of odd-odd nuclei, the best description was found for a $G_{0} / G_{1}$ ratio slightly larger than 1 [31]. However, our previous study on ${ }^{116} \mathrm{Cd}$ showed that
$G_{0} / G_{1}$ should not be much larger than 1 in order to reproduce the IAS and Gamow-Teller properties [17]. Therefore, we adopt our previous prescription of keeping $G_{0} / G_{1}=1$, which should be a reasonable parameter choice considering both types of data.

The HFB and pnQRPA equations are solved using a new version of the code HOSPHE [21].

\section{B. Charged-current neutrino-nucleus scattering}

We consider in this work the charged-current (anti)neutrinonucleus scattering. Therefore, we give in this section a brief summary of the main points of the formalism and we refer to $[15,32]$ for a more comprehensive treatment.

In a charged-current reaction a neutrino [antineutrino] is scattered from a nucleus $(A, Z)$ leading to a final nucleus $(A, Z+1)[(A, Z-1)]$ and an emitted lepton (antilepton):

$$
\begin{aligned}
& \nu_{l}+(A, Z) \rightarrow(A, Z+1)+l^{-}, \\
& \bar{v}_{l}+(A, Z) \rightarrow(A, Z-1)+l^{+},
\end{aligned}
$$

where $l$ stands for either an electron $(e)$, muon $(\mu)$ or tau $(\tau)$ lepton. These reactions proceed via the exchange of a charged $W^{+}$or $W^{-}$boson. In the case of the supernova neutrinos only the creation of an electron or a positron in the final state is possible due to the moderate energy $\left(E_{v} \lesssim 100 \mathrm{MeV}\right)$ of the incoming (anti)neutrino. In the present case we then have the transitions

$$
\begin{aligned}
& v_{e}+{ }^{A} \mathrm{~Pb} \rightarrow{ }^{A} \mathrm{Bi}+e^{-}, \\
& \bar{v}_{e}+{ }^{A} \mathrm{~Pb} \rightarrow{ }^{A} \mathrm{Tl}+e^{+},
\end{aligned}
$$

where $A=204,206,208$.

As mentioned, the energy of the impinging neutrino in our computations is low, and thus the transferred four-momentum is small compared to the mass of the exchanged charged boson, i.e., $Q^{2}=-q_{\mu} q^{\mu} \ll M_{W_{ \pm}}^{2}$. In this case the corresponding matrix element of the effective Hamiltonian can be written in the form

$$
\left\langle f\left|H_{\mathrm{eff}}\right| i\right\rangle=\frac{G}{\sqrt{2}} \int d^{3} \mathbf{r} l_{\mu} e^{-\mathrm{iq} \cdot \mathbf{r}}\left\langle f\left|\mathcal{J}^{\mu}(\mathbf{r})\right| i\right\rangle,
$$

where $\mathcal{J}^{\mu}(\mathbf{r})$ denotes the hadron current and $l_{\mu}$ is the lepton matrix element, $l_{\mu}=e^{\mathrm{iq} \cdot \mathbf{r}}\left\langle\ell\left|j_{\mu}(r)\right| \nu\right\rangle$ [15]. For the chargedcurrent processes the coupling constant is written as $G=$ $\cos \theta_{\mathrm{C}} G_{\mathrm{F}}$ where $G_{\mathrm{F}}$ is the Fermi coupling constant and $\theta_{\mathrm{C}}$ denotes the Cabibbo angle.

We assume in the present work that the final states $(f)$ and initial states $(i)$ have a well-defined angular momentum $J$ and parity $\pi$. Then, the double differential cross section for neutrino scattering from an initial state $J_{i}^{\pi_{i}}$ to a final state $J_{f}^{\pi_{f}}$ is given by

$$
\begin{aligned}
{\left[\frac{d^{2} \sigma_{i \rightarrow f}}{d \Omega d E_{\mathrm{exc}}}\right]_{v_{e} / \bar{v}_{e}}=} & \frac{G^{2}\left|\mathbf{k}^{\prime}\right| E_{\mathbf{k}^{\prime}}}{\pi\left(2 J_{i}+1\right)} F\left( \pm Z_{f}, E_{\mathbf{k}^{\prime}}\right) \\
& \times\left(\sum_{J \geqslant 0} \sigma_{\mathrm{CL}}^{J}+\sum_{J \geqslant 1} \sigma_{\mathrm{T}}^{J}\right),
\end{aligned}
$$


where $\mathbf{k}^{\prime}$ is the three-momentum, $E_{\mathbf{k}^{\prime}}$ is the energy of the outgoing electron or positron, and $F\left( \pm Z_{f}, E_{\mathbf{k}^{\prime}}\right)$ accounts for the distortion of the final-state electron $\left(+Z_{f}\right)$ or positron $\left(-Z_{f}\right)$ wave function by the Coulomb field of the final nucleus. Here $\sigma_{\mathrm{CL}}^{J}$ is the Coulomb-longitudinal component and $\sigma_{\mathrm{T}}^{J}$ is the transverse component defined as

$$
\begin{aligned}
\sigma_{\mathrm{CL}}^{J}= & (1+a \cos \theta)\left|\left(J_{f}\left\|\mathcal{M}_{J}(q)\right\| J_{i}\right)\right|^{2} \\
& +\left(1+a \cos \theta-2 b \sin ^{2} \theta\right)\left|\left(J_{f}\left\|\mathcal{L}_{J}(q)\right\| J_{i}\right)\right|^{2} \\
& +\frac{E_{\mathbf{k}}-E_{\mathbf{k}^{\prime}}}{q}(1+a \cos \theta+c) \\
& \times 2 \operatorname{Re}\left[\left(J_{f}\left\|\mathcal{L}_{J}(q)\right\| J_{i}\right)\left(J_{f}\left\|\mathcal{M}_{J}(q)\right\| J_{i}\right)^{*}\right],
\end{aligned}
$$

and

$$
\begin{aligned}
\sigma_{\mathrm{T}}^{J}= & \left(1-a \cos \theta+b \sin ^{2} \theta\right) \\
& \times\left[\left|\left(J_{f}\left\|\mathcal{T}_{J}^{\mathrm{mag}}(q)\right\| J_{i}\right)\right|^{2}+\left|\left(J_{f}\left\|\mathcal{T}_{J}^{\mathrm{el}}(q)\right\| J_{i}\right)\right|^{2}\right] \\
& \mp \frac{\left(E_{\mathbf{k}}+E_{\mathbf{k}^{\prime}}\right)}{q}(1-a \cos \theta-c) \\
& \times 2 \operatorname{Re}\left[\left(J_{f}\left\|\mathcal{T}_{J}^{\mathrm{mag}}(q)\right\| J_{i}\right)\left(J_{f}\left\|\mathcal{T}_{J}^{\mathrm{el}}(q)\right\| J_{i}\right)^{*}\right] .
\end{aligned}
$$

In the above expressions the minus sign refers to neutrino and the plus sign to antineutrino, and $E_{\mathbf{k}}$ is the energy of the incoming neutrino. Furthermore, we have introduced the notation

$$
\begin{gathered}
a=\sqrt{1-\frac{m_{f}^{2}}{E_{\mathbf{k}^{\prime}}^{2}},} \\
b=\frac{a^{2} E_{\mathbf{k}} E_{\mathbf{k}^{\prime}}}{q^{2}}, \\
c=\frac{m_{f}^{2}}{q E_{\mathbf{k}^{\prime}}},
\end{gathered}
$$

where the magnitude of the three-momentum transfer $q$ is given by

$$
q=|\mathbf{q}|=\sqrt{\left(E_{\mathbf{k}}-a E_{\mathbf{k}^{\prime}}\right)^{2}+2 a E_{\mathbf{k}} E_{\mathbf{k}^{\prime}}(1-\cos \theta)} .
$$

The definition of the operators $\mathcal{T}_{J M}=\mathcal{M}_{J M}, \mathcal{L}_{J M}, \mathcal{T}_{J M}^{\mathrm{el}}$, $\mathcal{T}_{J M}^{\mathrm{mag}}$ is given in [32]. In general, these operators contain both vector and axial-vector pieces, i.e., $\mathcal{T}_{J M}=T_{J M}^{\mathrm{V}}-T_{J M}^{\mathrm{A}}$. They depend on the nuclear form factors $F_{1,2}^{\mathrm{V}}\left(Q^{2}\right)$ (vector), $F^{\mathrm{A}}\left(Q^{2}\right)$ (axial-vector), and $F^{\mathrm{P}}\left(Q^{2}\right)$ (pseudoscalar), which depend on the four-momentum transfer $Q^{2}=-q_{\mu} q^{\mu}$ [15]. For small momentum transfers the cross sections are typically dominated by Gamow-Teller-like transitions mediated by the operator $F^{\mathrm{A}}(q) j_{0}(q r) \sigma$ and Fermi-like ones which proceed via the operator $F^{\mathrm{V}}(q) j_{0}(q r) \mathbf{1}$. Additionally, for supernova neutrinos, the spin-dipole-like transitions of the form $F^{\mathrm{A}}(q)\left[j_{1}(q r) \boldsymbol{Y}_{1} \sigma\right]_{0^{-}, 1^{-}, 2^{-}}$turn out to be important.

For the neutrino and antineutrino scattering we have adopted the quenched static value $F^{A}(0)=-1.00$ and the axial-vector mass $M_{\mathrm{A}}=1016 \mathrm{MeV}$. This is in agreement with the fact that the charge-exchange experiments measure only $60-70 \%$ of the GT strength given by the Ikeda sum rule [33]. It is also in agreement with the determined quenching of the
TABLE I. Computed centroids of the Gamow-Teller giant resonance relative to the ground state in the $\mathrm{Bi}$ nuclei. The values on the left-hand side are the results of our computation with $G_{1}=G_{0}=G_{n}$ and on the right hand side for $G_{1}=G_{0}=G_{p}$.

\begin{tabular}{lccc}
\hline \hline & ${ }^{204} \mathrm{~Pb}$ & ${ }^{206} \mathrm{~Pb}$ & ${ }^{208} \mathrm{~Pb}$ \\
\hline $\mathrm{SkM}^{*}$ & $14.60,14.61$ & $15.32,15.21$ & $16.43,16.37$ \\
$\mathrm{SkX}$ & $12.00,11.97$ & $12.56,12.47$ & $13.74,13.71$ \\
SLy4 & $15.23,15.19$ & $15.94,15.34$ & $16.90,16.84$ \\
Expt. & & & $15.23 \mathrm{MeV} \mathrm{[39]}$ \\
\hline \hline
\end{tabular}

Gamow-Teller strength stemming from non-nucleonic degrees of freedom [34] and based on shell-model calculations [35,36].

At this point it is appropriate to note the treatment of the final-state Coulomb effects, represented by the Fermi function $F\left( \pm Z_{f}, E_{\mathbf{k}^{\prime}}\right)$ in (6). The distortion is to be treated differently in the regions of small and large values of the so-called effective momentum

$$
k_{\mathrm{eff}}=\sqrt{E_{\mathrm{eff}}^{2}-m_{e^{ \pm}}^{2}}
$$

where $m_{e^{+}}\left(m_{e^{-}}\right)$is the positron (electron) mass and the effective energy is given by

$$
E_{\text {eff }}=E_{\mathbf{k}^{\prime}}-V_{\mathrm{C}}(0) \text {. }
$$

Here $V_{\mathrm{C}}(0)$ is the Coulomb potential at the center of the final nucleus. For small values of $k_{\text {eff }}$ we use the Fermi function but for large values of $k_{\text {eff }}$ we adopt the so-called modified effective momentum approximation (MEMA), introduced in [37]. Consequently, for large $k_{\text {eff }}$ we drop the Fermi function from (6) and, instead, replace the absolute value of the threemomentum and the energy of the outgoing electron/positron by their effective values (13) and (14). For more details see [15].

\section{RESULTS}

The charged-current cross sections of the (anti)neutrino scattering off the stable even-even lead isotopes have been computed in this study, at energies typical of supernova neutrinos. The charge-changing quasiparticle random-phase approximation (pnQRPA) has been adopted for the nuclearstructure description, with the two-body interaction taken to be of the Skyrme type. The considered Skyrme parametrizations in this study are SkM*, SkX, and SLy4. The adopted values of

TABLE II. Computed GTGR $\beta^{-}$Gamow-Teller strengths $S^{-}$(GTGR) and the measured one for ${ }^{208} \mathrm{~Pb}$. The results on the left are those for $G_{1}=G_{0}=G_{n}$ and on the right those for $G_{1}=G_{0}=G_{p}$. Both choices of pairing give exactly the same GTGR strength for ${ }^{208} \mathrm{~Pb}$. The column "normalized" gives the computed numbers of column 3 multiplied by the experimental quenching 0.56 .

\begin{tabular}{lcccc}
\hline \hline & ${ }^{204} \mathrm{~Pb}$ & ${ }^{206} \mathrm{~Pb}$ & ${ }^{208} \mathrm{~Pb}$ & ${ }^{208} \mathrm{~Pb}$ (normalized) \\
\hline $\mathrm{SkM}^{*}$ & $81.39,82.92$ & $82.79,87.05$ & 91.93 & 51.48 \\
SkX & $75.22,80.59$ & $83.69,85.45$ & 89.98 & 50.39 \\
SLy4 & $87.0,86.64$ & $92.05,92.97$ & 98.21 & 55.0 \\
Expt. & & & & $60.84 \pm 13.67[39]$
\end{tabular}


TABLE III. Total $\beta^{-}$and $\beta^{+}$GT strengths $S^{\mp}$ of Eq. (15). The values on the left (right) are the results obtained with pairing stength $G_{n}\left(G_{p}\right)$. The total strength for ${ }^{208} \mathrm{~Pb}$ is independent of the pairing strength.

\begin{tabular}{llccc}
\hline \hline & & ${ }^{204} \mathrm{~Pb}$ & ${ }^{206} \mathrm{~Pb}$ & ${ }^{208} \mathrm{~Pb}$ \\
\hline SkM $^{*}$ & $\beta^{-}$ & $124.03,123.42$ & $128.95,128.64$ & 134.09 \\
& $\beta^{+}$ & $3.99,3.38$ & $2.92,2.61$ & 2.06 \\
$\mathrm{SkX}$ & $\beta^{-}$ & $124.38,123.94$ & $129.17,128.94$ & 134.13 \\
& $\beta^{+}$ & $4.34,3.90$ & $3.13,2.89$ & 2.08 \\
SLy4 & $\beta^{-}$ & $123.41,123.03$ & $128.61,128.24$ & 133.93 \\
& $\beta^{+}$ & $3.36,2.98$ & $2.56,2.19$ & 1.88 \\
\hline \hline
\end{tabular}

the pairing strength in these computations are those presented in [38]. In [38] there are two values for the separable Gaussian pairing, labeled $G_{n}$ and $G_{p}$. In the present pnQRPA calculations the results are affected by both $G_{0}$ and $G_{1}$ of the separable Gaussian pairing, present at the HFB level. The strengths $G_{n}$ and $G_{p}$ of [38] relate to $G_{1}$ only so we chose to use both strengths in two separate pnQRPA calculations, one with $G_{1}=G_{p}$ and one with $G_{1}=G_{n}$, to see the effect of pairing on the scattering observables. The value of $G_{0}$ is not determined at the HFB level without proton-neutron mixing but a reasonable choice is $G_{0}=G_{1}$, adopted also in our previous study for ${ }^{116} \mathrm{Cd}[17]$.
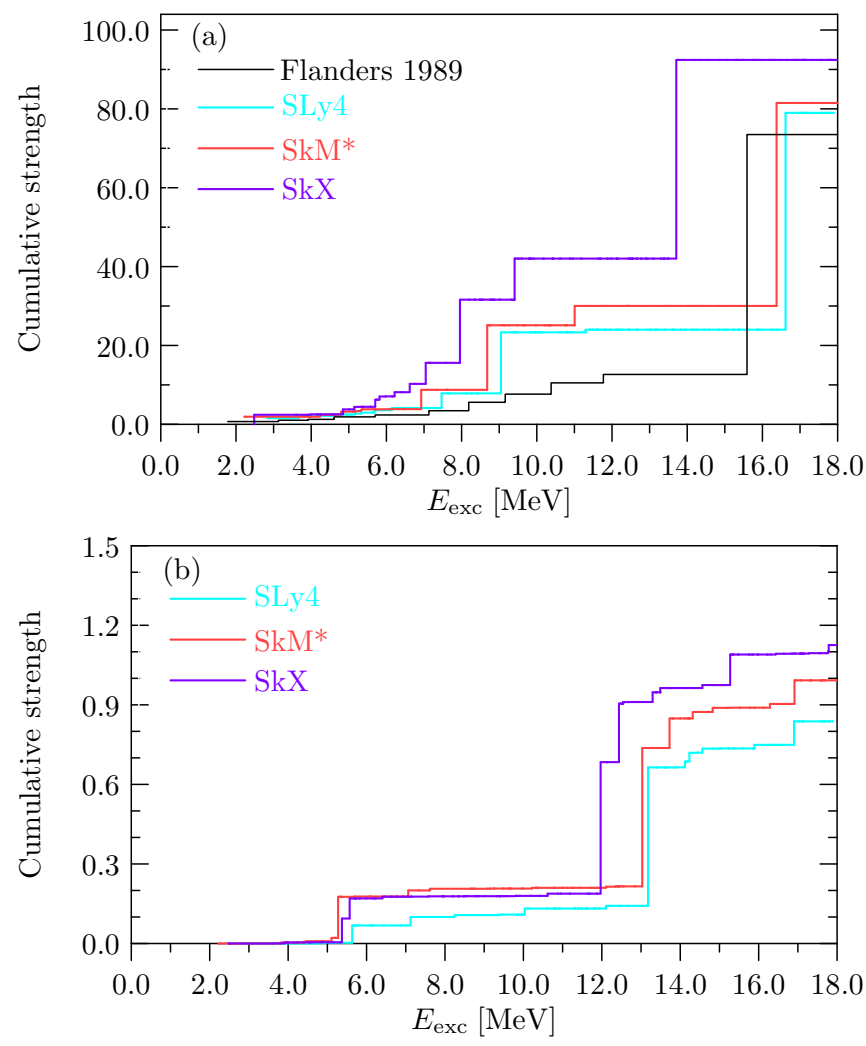

FIG. 1. Cumulative $\beta^{-}$(a) and $\beta^{+}$(b) strengths for Gamow-Teller transitions from ${ }^{208} \mathrm{~Pb}$ to states in ${ }^{208} \mathrm{Bi}$ and ${ }^{208} \mathrm{Tl}$ computed using the three interactions. Flanders 1989 refers to Ref. [39].
TABLE IV. Positions of the IAS. The left and right values correspond to the results with pairing strength $G_{n}$ and $G_{p}$, respectively. The energies in this table are measured relative to the ground state of lead isotope.

\begin{tabular}{lccc}
\hline \hline & ${ }^{204} \mathrm{~Pb}$ & ${ }^{206} \mathrm{~Pb}$ & ${ }^{208} \mathrm{~Pb}$ \\
\hline $\mathrm{SkM}^{*}$ & $14.71,14.72$ & $15.00,14.90$ & $15.49,15.43$ \\
SkX & $17.72,17.73$ & $18.01,17.92$ & $18.62,18.66$ \\
SLy4 & $16.26,16.21$ & $16.49,15.86$ & $16.79,16.73$ \\
Expt. & & & $17.47 \mathrm{MeV} \mathrm{[39]}$ \\
\hline \hline
\end{tabular}

\section{A. Properties of the various isovector excitations}

We begin by analyzing the spin-isospin and isospin properties of the studied lead nuclei since experimental data are available for comparison. We start by a discussion of the Gamow-Teller giant resonance (GTGR) in the bismuth nuclei. The calculated energy centroids of the $\beta^{-}$GTGR for the discussed lead isotopes are shown in Table I. The experimental resonance energy for ${ }^{208} \mathrm{~Pb}$ [39] is also shown in Table I. Unfortunately no experimental data are available for the other considered lead nuclei. The two values of a computed energy centroid in Table I come from the two adopted values of the pairing strength, one for $G_{1}=G_{0}=G_{n}$ and the other for $G_{1}=G_{0}=G_{p}$. Our computed centroids for ${ }^{208} \mathrm{~Pb}$ are shifted
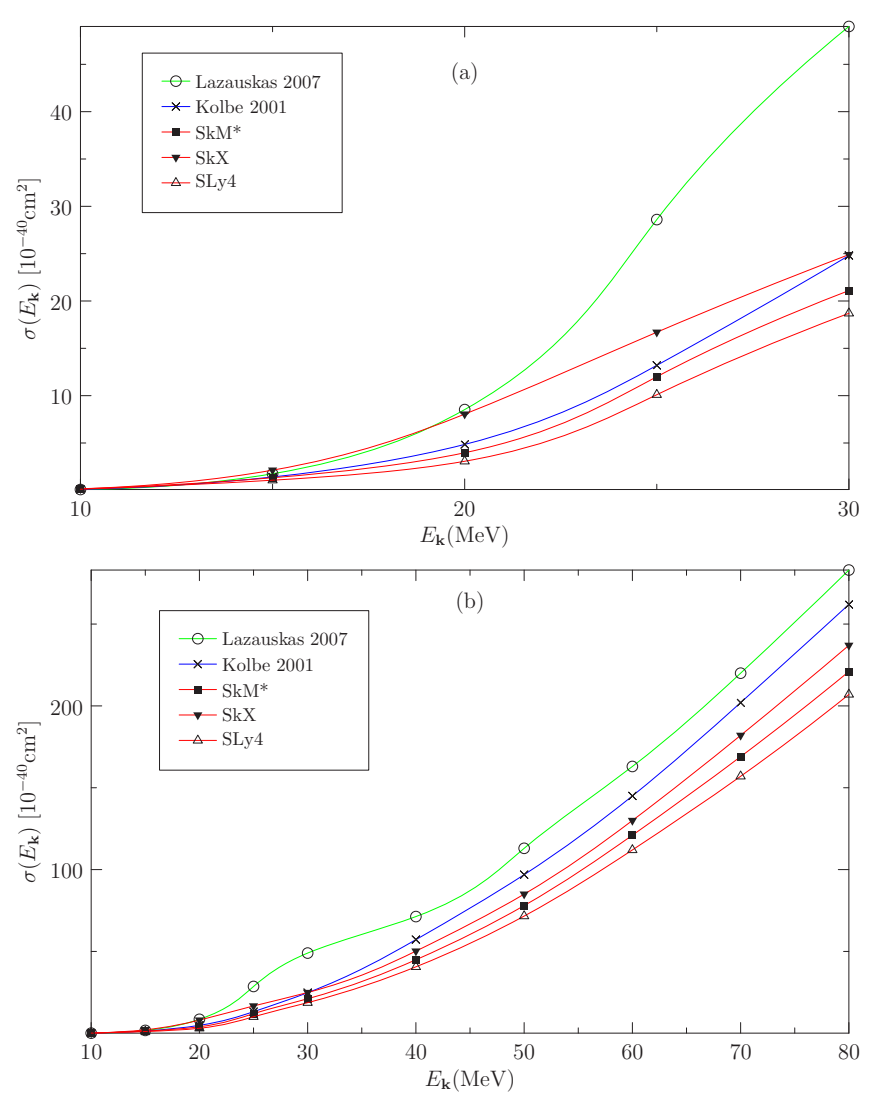

FIG. 2. Comparison of the computed total CC neutrino cross sections as functions of the energy of the incoming neutrino for ${ }^{208} \mathrm{~Pb}$. The upper panel (a) is a magnification of the low-energy region of the lower panel (b). In this figure Lazauskas 2007 refers to Ref. [25] and Kolbe 2001 refers to Ref. [24] 
TABLE V. Average energies for $\beta^{-}$and $\beta^{+}$transitions to $J^{\pi}$ with $L=1,2$ for ${ }^{208} \mathrm{~Pb}$. The energies are in $\mathrm{MeV}$ and measured relative to the ground state of ${ }^{208} \mathrm{~Pb}$. The values on the left (right) correspond to the pairing strength $G_{n}\left(G_{p}\right)$.

\begin{tabular}{ccccccc}
\hline \hline & $L$ & $J^{\pi}$ & SkM $^{*}$ & SkX & SLy4 & Ref. [43] \\
\hline$\beta^{-}$ & 1 & $0^{-}$ & $29.99,29.93$ & $26.42,26.38$ & $31.46,31.40$ & 32.1 \\
& & $1^{-}$ & $26.55,26.49$ & $23.84,23.80$ & $28.10,28.04$ & 29.5 \\
& & $2^{-}$ & $21.00,20.93$ & $19.05,19.02$ & $22.30,22.24$ & 25.1 \\
& 2 & $1^{+}$ & $31.88,31.82$ & $29.18,29.14$ & $33.22,33.16$ & 36.8 \\
& $2^{+}$ & $28.63,28.57$ & $26.92,26.89$ & $30.06,30.00$ & 34.7 \\
& $3^{+}$ & $23.80,23.74$ & $23.17,23.14$ & $25.27,25.21$ & 28.9 \\
$\beta^{+}$ & 1 & $0^{-}$ & $13.91,13.84$ & $12.36,12.32$ & $12.79,12.73$ & 12.5 \\
& & $1^{-}$ & $13.08,13.02$ & $12.15,12.11$ & $11.99,11.93$ & 7.6 \\
& $2^{-}$ & $13.54,13.48$ & $13.39,13.35$ & $13.12,13.06$ & 14.4 \\
& 2 & $1^{+}$ & $21.14,21.08$ & $19.22,19.19$ & $21.11,21.06$ & 20.2 \\
& $2^{+}$ & $16.65,16.58$ & $15.88,15.84$ & $16.89,16.83$ & 13.8 \\
& $3^{+}$ & $13.02,12.96$ & $13.47,13.44$ & $13.62,13.56$ & 13.3 \\
\hline \hline
\end{tabular}

up for $\mathrm{SkM}^{*}$ and SLy4 and down for SkX as compared with the experimental centroid at $15.23 \mathrm{MeV}$ [39]. The shift up for the SLy4 interaction is higher than for the $\mathrm{SkM}^{*}$ interaction. For the three lead nuclei the two extreme centroids always stem from SkX and SLy4, the centroid predicted by $\mathrm{SkM}^{*}$ being in between, close to the SLy4 prediction. The theoretical GTGR is mainly a neutron $0 i_{13 / 2}$ to proton $0 i_{11 / 2}$ transition.

Table II shows the computed $\beta^{-}$GTGR strengths for the spin-isospin operator. This strength is part of the total strength defined as

$$
S^{ \pm}=\sum_{f}\left|\left(1_{f}^{+}\left\|\sum_{k=1}^{A} \boldsymbol{\sigma}_{k} \cdot t_{k}^{ \pm}\right\| 0_{\mathrm{gs}}^{+}\right)\right|^{2}
$$

over the $1^{+}$final states, $1_{f}^{+}$, of the involved odd-odd nucleus. Here $t_{k}^{ \pm}$is the isospin raising (lowering) operator for the $\beta^{-}\left(\beta^{+}\right)$branch. In this way the total $\beta^{-}$and $\beta^{+}$strengths satisfy the Ikeda sum rule $S^{-}-S^{+}=3(N-Z)$, as do also our present calculations. For the GTGR strength, $S^{-}$(GTGR) the sum runs over final states which constitute the GTGR. It is seen in Table II that the computed strengths for ${ }^{208} \mathrm{~Pb}$ are inside the measured interval $60.84 \pm 13.67$ [39] for all the considered Skyrme interactions. Our two computations $\left(G_{1}=G_{0}=G_{n}\right.$ and $\left.G_{1}=G_{0}=G_{p}\right)$ give exactly the same GTGR strength for ${ }^{208} \mathrm{~Pb}$. It is worth mentioning that the measurements performed in [39] yield only 56\% of the Ikeda sum rule. Therefore, our computed values in Table II, last column (labeled "normalized") have been multiplied by a factor of 0.56 for ${ }^{208} \mathrm{~Pb}$ to allow quantitative comparison with the data.

TABLE VI. Total cross sections for the charged-current neutrino scatterings in units of $10^{-42} \mathrm{~cm}^{2}$. The values on the left correspond to our computation with pairing strength $G_{n}$ and on the right with $G_{p}$. Only one value is present in cases for which the difference between the two obtained values is less than $0.05 \%$. The numbers in parentheses refer to exponents.

\begin{tabular}{|c|c|c|c|c|c|c|}
\hline \multirow[t]{2}{*}{$E_{\mathbf{k}} / \mathrm{MeV}$} & \multicolumn{3}{|c|}{${ }^{204} \mathrm{~Pb}$} & \multicolumn{3}{|c|}{${ }^{206} \mathrm{~Pb}$} \\
\hline & $\mathrm{SkM}^{*}$ & SkX & SLy4 & $\mathrm{SkM}^{*}$ & SkX & SLy4 \\
\hline 5.0 & $(2.55,2.02)(-6)$ & $(2.91,3.21)(-7)$ & $(5.68,8.11)(-7)$ & $(1.36,2.83)(-1)$ & $(4.22,5.52)(-6)$ & $(3.06,8.04)(-6)$ \\
\hline 10.0 & $(1.45,1.35) \quad(1)$ & $(1.86)$ & $(1.04,1.07) \quad(1)$ & $(1.60,1.67)$ & $(2.11,2.25) \quad(1)$ & $(1.16,1.69) \quad(1)$ \\
\hline 15.0 & $(1.34,1.28) \quad(2)$ & $(2.18,2.16) \quad(2)$ & $(1.05,1.06)$ & $(1.38,1.40) \quad$ (2) & $(2.28,2.32) \quad(2)$ & $(1.08,1.37)$ \\
\hline 20.0 & $(4.15,4.00) \quad(2)$ & $(8.00,7.94)$ & (3.15) (2) & $(4.21,4.25)$ & $(8.34,8.46) \quad(2)$ & $(3.13,3.62)$ \\
\hline 25.0 & $(1.20,1.17)$ & $(1.62,1.61)$ & (1.00) (3) & $(1.22,1.23) \quad$ (3) & $(1.68,1.69) \quad$ (3) & $(1.01,1.09) \quad$ (3) \\
\hline 30.0 & $(2.07,2.03) \quad$ (3) & $(2.41,2.40)$ & (1.81) (3) & $(2.12) \quad(3)$ & $(2.49,2.50) \quad$ (3) & $(1.84,1.92)$ \\
\hline 40.0 & $(4.39,4.32) \quad(3)$ & $(4.88,4.86) \quad(3)$ & (3.91) (3) & $(4.47) \quad(3)$ & $(5.01,5.03) \quad(3)$ & $(4.00,4.09) \quad(3)$ \\
\hline 50.0 & $(7.66,7.51)$ & $(8.24,8.22)$ & $(6.88,6.89) \quad(3)$ & (7.73) (3) & $(8.45,8.47) \quad(3)$ & $(7.03,7.15) \quad(3)$ \\
\hline 60.0 & $(1.19,1.16)$ & (1.26) & (1.08) (4) & (1.19) (4) & $(1.29)$ & $(1.10,1.11)$ \\
\hline 70.0 & $(1.66,1.63)$ & $(1.77,1.76)$ & (1.52) (4) & (1.67) (4) & (1.80) (4) & $(1.55,1.56)$ \\
\hline 80.0 & $(2.18,2.14) \quad$ (4) & $(1.20) \quad(4)$ & (2.00) (4) & (1.19) (4) & (1.35) (4) & $(2.03,2.05) \quad$ (4) \\
\hline \multirow[t]{2}{*}{$E_{\mathbf{k}} / \mathrm{MeV}$} & & \multicolumn{5}{|c|}{${ }^{208} \mathrm{~Pb}$} \\
\hline & & $\mathrm{SkM}^{*}$ & & SkX & & SLy4 \\
\hline 5.0 & & $(2.3 f 2,2.85)(-5)$ & & $(4.73,5.20)(-6)$ & & $(0.92,1.01)(-5)$ \\
\hline 10.0 & & $(1.49,1.54) \quad(1)$ & & $(1.64,1.75) \quad(1)$ & & $(1.20,1.23)$ \\
\hline 15.0 & & $(1.33,1.35)$ & & $(2.13,2.15) \quad(2)$ & & $(1.07,1.09) \quad$ (2) \\
\hline 20.0 & & $(3.97,4.04) \quad(2)$ & & $(8.05,8.11)$ & & $(3.07,3.12)$ \\
\hline 25.0 & & $(1.20,1.21)$ & & $(1.67,1.68) \quad(3)$ & & $(1.01,1.03) \quad$ (3) \\
\hline 30.0 & & $(2.11,2.12)$ & & (2.49) (3) & & $(1.87,1.88)$ \\
\hline 40.0 & & $(4.49,4.51)$ & & $(5.02,5.03)$ & & $(4.06,4.08)$ \\
\hline 50.0 & & $(7.80,7.83)$ & & $(8.50,8.52)$ & & $(7.16,7.18)$ \\
\hline 60.0 & & $(1.21)$ & & $(1.30)$ & & (1.12) (4) \\
\hline 70.0 & & (1.69) & & (1.82) (4) & & $(1.57,1.58)$ \\
\hline 80.0 & & $(2.21)$ & & (2.37) (4) & & (2.07) (4) \\
\hline
\end{tabular}


TABLE VII. Same as Table VI for antineutrino scattering.

\begin{tabular}{|c|c|c|c|c|c|c|}
\hline$E_{\mathbf{k}} / \mathrm{MeV}$ & \multicolumn{3}{|c|}{${ }^{204} \mathrm{~Pb}$} & \multicolumn{3}{|c|}{${ }^{206} \mathrm{~Pb}$} \\
\hline 10.0 & $(2.74,1.91)(-1)$ & $(2.86,2.39)(-1)$ & $(2.17,1.81)(-1)$ & $(9.03,6.70)(-2)$ & $(9.72,8.18)(-2)$ & $(7.42,4.94)(-2)$ \\
\hline 15.0 & $(1.30,1.04) \quad(0)$ & $(1.46,1.30) \quad(0)$ & $(1.19,1.06) \quad(0)$ & $(6.42,5.48)(-1)$ & $(7.89,7.23)(-1)$ & $(5.99,4.31)(-1)$ \\
\hline 20.0 & $(3.93,3.43) \quad(0)$ & $(4.15,3.84) \quad(0)$ & $(3.77,3.49) \quad(0)$ & $(2.43,2.22)$ & $(2.65,2.51) \quad(0)$ & $(2.35,1.92) \quad(0)$ \\
\hline 40.0 & $(4.61,4.39)$ & $(4.49,4.34)$ & $(4.50,4.36) \quad(1)$ & $(3.72,3.61)$ & $(3.61,3.53)$ & $(3.60,3.30)$ \\
\hline 50.0 & $(1.25,1.20)$ & $(1.24,1.21)$ & $(1.24,1.20) \quad$ (2) & $(1.01,0.98)$ & $(1.01,1.99)$ & $(9.89,8.98)$ \\
\hline 60.0 & $(3.15,3.03)$ & $(3.12,3.04)$ & $(3.08,3.00) \quad(2)$ & $(2.66,2.59)$ & $(2.64,2.60)$ & $(2.57,2.35)$ \\
\hline 70.0 & $(6.61,6.42)$ & $(6.56,6.43)$ & $(6.48,6.34) \quad(2)$ & $(5.85,5.73)$ & $(5.80,5.72)$ & $(5.66,5.27)$ \\
\hline 80.0 & $(1.17,1.15)$ & $(1.17,1.15)$ & $(1.16,1.14) \quad(3)$ & $(1.07,1.05)$ & $(1.07,1.05)$ & $(1.04,0.99) \quad$ (3) \\
\hline 15.0 & & $(2.26,2.32)(-1)$ & & $(3.23,3.27)(-1)$ & & $(3.18,3.25)(-1)$ \\
\hline 20.0 & & $(1.35,1.37)$ & & $(1.48,1.49) \quad(0)$ & & $(1.60,1.61) \quad(0)$ \\
\hline 25.0 & & $(4.10,4.13)$ & & $(4.13,4.14)$ & & $(4.54,4.58)$ \\
\hline 30.0 & & $(9.27,9.33)$ & & $(8.96,8.99)$ & & $(9.92,9.98)$ \\
\hline 40.0 & & $(3.02,3.03)$ & & $(2.85,2.86)$ & & $(3.13,3.14)$ \\
\hline 50.0 & & $(8.20,8.25)$ & & $(8.00,8.02)$ & & $(8.68,8.73)$ \\
\hline 60.0 & & $(2.28,2.30)$ & & $(2.21,2.22)$ & & $(2.37,2.38)$ \\
\hline 70.0 & & $(5.26,5.28)$ & & $(5.10,5.11)$ & & $(5.40,5.42)$ \\
\hline 80.0 & & $(9.88,9.91)$ & & $(9.67,9.68)$ & & $(1.01,1.01)$ \\
\hline
\end{tabular}

The total GT strengths for the $\beta^{-}$and $\beta^{+}$transitions are presented in Table III. The total GT strengths for $\beta^{+}$are very small as compared with $\beta^{-}$. The Ikeda sum rule is satisfied in our computations. In Fig. 1, panels (a) and (b), we show the cumulative $\beta^{-}$and $\beta^{+}$strengths for Gamow-Teller transitions from ${ }^{208} \mathrm{~Pb}$ to ${ }^{208} \mathrm{Bi}$ and ${ }^{208} \mathrm{Tl}$, respectively. For the $\beta^{-}$GT strength there are experimental data available [39] to compare with. As seen in panel (a) the experimental cumulative sum is qualitatively reproduced by the $\mathrm{SkM}^{*}$ and SLy4 interactions, with the SkX interaction predicting excessive strength at low energies due to too low an energy of the GTGR (see Table I). Even the $\mathrm{SkM}^{*}$ and SLy4 interactions cannot reproduce quantitatively the $\beta^{-}$strength below the GTGR. None of the used interactions has been fitted to spin-isospin properties of nuclei, so it is natural to expect deviations from the experimental strength distribution. It should be noted that the theoretical GTGR strengths in panel (a) have been multiplied by the experimental quenching 0.56 [39] to allow quantitative comparison. In the case of the $\beta^{+}$strength [panel (b)] the three interactions predict a similar qualitative behavior of the strength - the strength saturating quite high, around $16 \mathrm{MeV}$-for the three interactions. For all three interactions the main contribution comes from around $12-13 \mathrm{MeV}$, from a proton $0 h_{11 / 2}$ to neutron $0 h_{9 / 2}$ transition in the calculations.

The computed positions of the isobaric analog states for the discussed lead isotopes are shown in Table IV. The energies in Table IV are computed relative to the ground state of lead isotope as follows:

$$
E_{\mathrm{exc}}^{\prime}= \begin{cases}E_{\mathrm{exc}}+Q_{\mathrm{EC}}-m_{e} c^{2} & \text { for } \beta^{-} \text {strength, } \\ E_{\mathrm{exc}}+Q_{\beta^{-}}+m_{e} c^{2} & \text { for } \beta^{+} \text {strength, }\end{cases}
$$

where $E_{\mathrm{exc}}^{\prime}$ is the excitation energy relative to the ground state of the lead isotopes and $E_{\text {exc }}$ is the excitation energy relative to the ground state of the daughter nucleus. The $Q$ values, $Q_{\beta^{-}}$and $Q_{\mathrm{EC}}$, are adopted from [40]. The computed positions of the IAS in ${ }^{208} \mathrm{Bi}$ are shifted down for the SkM* and SLy4 interactions while the IAS is shifted up for the SkX interaction as compared with the experimental position [39]. For the three nuclei the computed energy of the IAS varies by $3 \mathrm{MeV}$, with SkX giving the highest and the $\mathrm{SkM}^{*}$ giving the lowest values. The energies predicted by SLy4 are roughly halfway between the values given by the other two interactions. In general, the impact of the adopted values of the pairing strength $\left(G_{n}, G_{p}\right)$ on the properties of the GTGR and IAS are very small.

The deviations of the computed locations of the IAS from the experimental one are 4\% for SLy4, 6\% for SkX, and 11\% for $\mathrm{SkM}^{*}$. The deviations are explained by the different ways of fitting the parameters of the different Skyrme forces. The SkX was effectively implemented by leaving out the Coulomb exchange part of the Hamiltonian. This explains why the SkX results deviate from the results of the other two interactions. The results of the other two interactions, SkM* and SLy4, deviate from each other by some $1.5 \mathrm{MeV}$. This deviation can be compared with the corresponding one obtained in the 

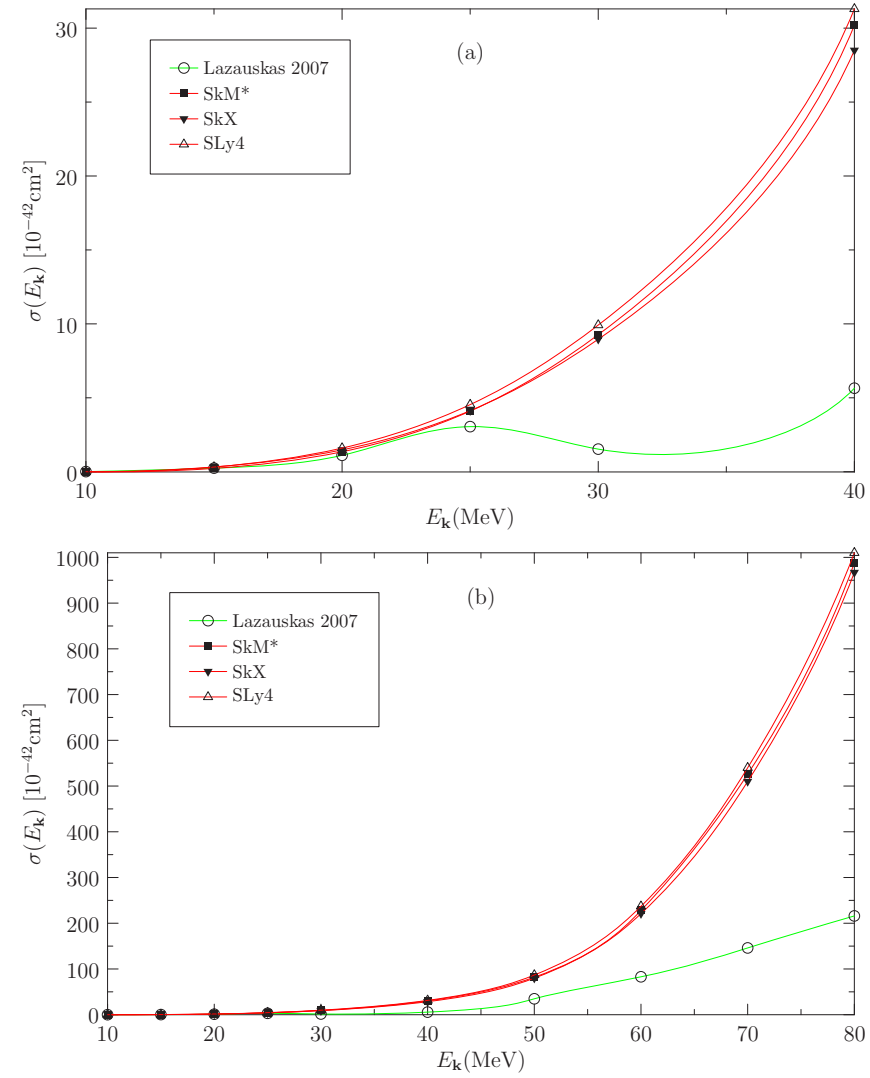

FIG. 3. Comparison of the computed total $\mathrm{CC}$ antineutrino cross sections as functions of the energy of the incoming antineutrino for ${ }^{208} \mathrm{~Pb}$. The upper panel (a) is a magnification of the low-energy region of the lower panel (b). Lazauskas 2007: Ref. [25]. analysis of the location of the IAR (isobaric analog resonance) for the $\mathrm{Zr}$ isotopes in [41]. The calculations of [41] and ours are very comparable since in both calculations the IAR is just one peak, without notable fragmentation. As can be seen in Fig. 2 of [41], the locations of the IAR for the two interactions, $\mathrm{SkM}^{*}$ and SLy4, deviate by some $2 \mathrm{MeV}$ throughout the $\mathrm{Zr}$ chain $A=98-112$. This is even more than for the presently studied three stable $\mathrm{Pb}$ isotopes. A more recent study of ${ }^{208} \mathrm{~Pb}$, using different SAMi-J Skyrme functionals, records similar fluctuations in the location of the IAS depending on the adopted symmetry energy of the functional at the saturation density of nuclear matter [42]. The present study and the other two, quoted above, show that the location of the IAS varies by a few $\mathrm{MeV}$ depending on the adopted Skyrme parametrization.

For completeness we computed the average energies for the isovector spin-dipole $0^{-}, 1^{-}$, and $2^{-}$strengths with $L=1$ and for the isovector spin-quadrupole $1^{+}, 2^{+}$, and $3^{+}$strengths with $L=2$ at the limit of zero momentum transfer. We present our results in Table $\mathrm{V}$. We compare our average excitation energies for ${ }^{208} \mathrm{~Pb}$ with the ones of Ref. [43]. We achieve a good agreement with the aforementioned reference. For $\beta^{-}$transition, the results obtained by the SLy4 interaction have the best agreement with the results of Ref. [43]. The SkX interaction predicts lower average energies than the other interactions except for $\beta^{+}$transition to $1^{-}, 2^{-}$, and $3^{+}$. Our average energies for the $\beta^{+}$transitions to $1^{-}$and $2^{+}$are slightly shifted up compared to Ref. [43].

\section{B. Total cross sections}

The total cross sections for the charged-current (anti)neutrino scattering off the even-even stable lead isotopes have been computed using the formalism outlined in Sec. II B.

TABLE VIII. Average cross sections for the charged-current neutrino scattering in units of $10^{-42} \mathrm{~cm}^{2}$. The values on the left (right) correspond to computations with pairing strength $G_{n}\left(G_{p}\right)$. Only one value is present for which the difference between the two obtained values is less than $0.05 \%$. The values in the columns "Full range" run from the smallest to the largest value predicted by the three considered Skyrme interactions. The numbers in parentheses refer to exponents.

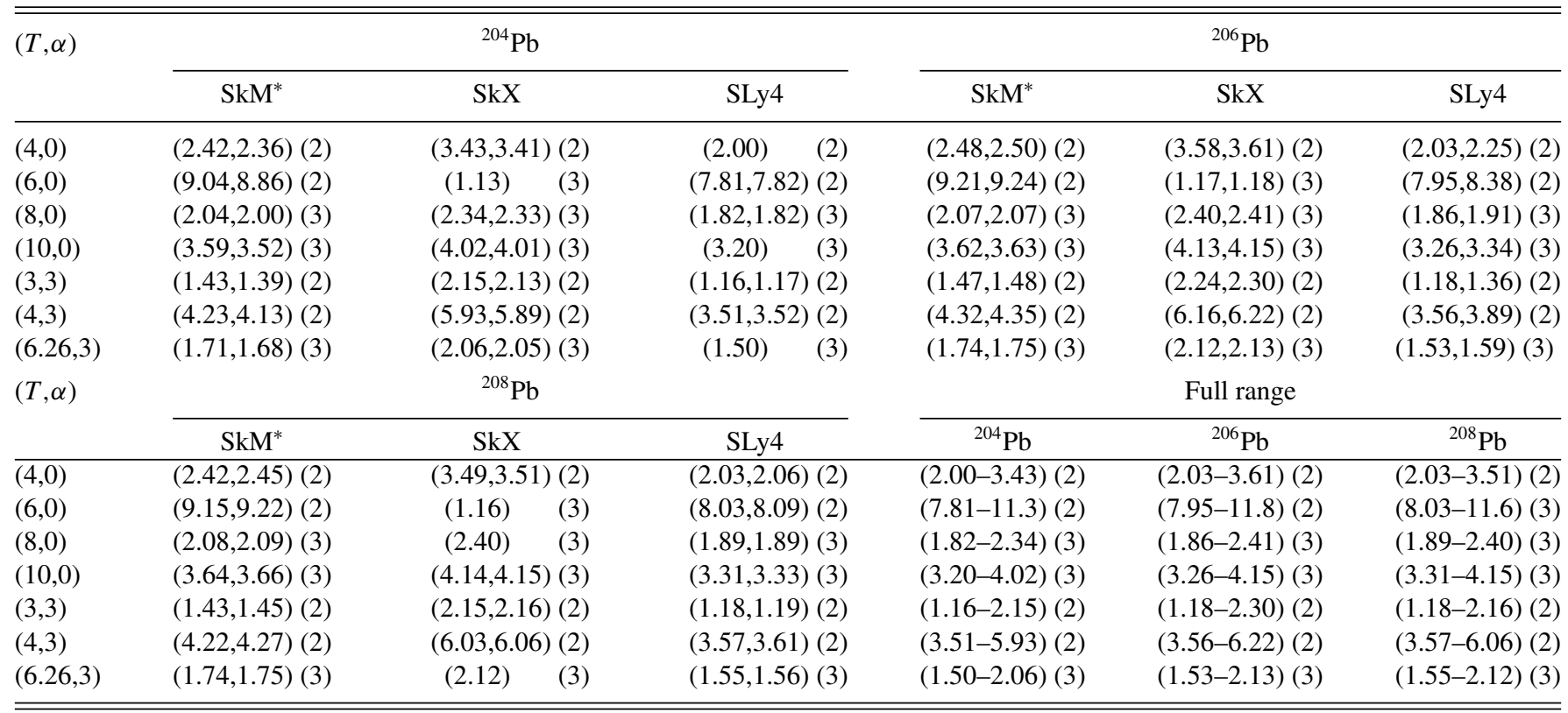


TABLE IX. Same as Table VIII for antineutrino scattering.

\begin{tabular}{|c|c|c|c|c|c|c|}
\hline \multirow[t]{2}{*}{$(T, \alpha)$} & \multicolumn{3}{|c|}{${ }^{204} \mathrm{~Pb}$} & \multicolumn{3}{|c|}{${ }^{206} \mathrm{~Pb}$} \\
\hline & $\mathrm{SkM}^{*}$ & SkX & SLy4 & $\mathrm{SkM}^{*}$ & SkX & SLy4 \\
\hline$(6,0)$ & $(1.10,1.03)(1)$ & $(1.10,1.05)(1)$ & $(1.07,1.03)(1)$ & $(8.55,8.23)(0)$ & $(8.56,8.34)(0)$ & $(8.30,7.46)(0)$ \\
\hline$(8,0)$ & $(3.71,3.55)(1)$ & $(3.68,3.58)(1)$ & $(3.64,3.54)(1)$ & $(3.10,3.02)(1)$ & $(3.09,3.03)(1)$ & $(3.02,2.77)(1)$ \\
\hline$(10,0)$ & $(9.52,9.22)(1)$ & $(9.48,9.27)(1)$ & $(9.39,9.18)(1)$ & $(8.31,8.13)(1)$ & $(8.28,8.16)(1)$ & $(8.11,7.57)(1)$ \\
\hline$(6.26,3)$ & $(2.28,2.16)(1)$ & $(2.27,2.19)(1)$ & $(2.24,2.16)(1)$ & $(1.82,1.76)(1)$ & $(1.82,1.77)(1)$ & $(1.77,1.61)(1)$ \\
\hline \multirow[t]{2}{*}{$(T, \alpha)$} & \multicolumn{3}{|c|}{${ }^{208} \mathrm{~Pb}$} & \multicolumn{3}{|c|}{ Full range } \\
\hline & $\mathrm{SkM}^{*}$ & SkX & SLy4 & ${ }^{204} \mathrm{~Pb}$ & ${ }^{206} \mathrm{~Pb}$ & ${ }^{208} \mathrm{~Pb}$ \\
\hline$(4,0)$ & $(0.99,1.00)(0)$ & $(1.01,1.02)(0)$ & $(1.10,1.11)(0)$ & $(2.02-2.30)(0)$ & $(1.25-1.58)(0)$ & $(0.99-1.11)(0)$ \\
\hline$(6,0)$ & $(6.69,6.73)(0)$ & $(6.53,6.55)(0)$ & $(7.07,7.11)(0)$ & $(1.03-1.10)(1)$ & $(7.46-8.56)(0)$ & $(6.53-7.11)(0)$ \\
\hline$(4,3)$ & $(1.82,1.83)(0)$ & $(1.83,1.84)(0)$ & $(1.99,2.01)(0)$ & $(3.51-3.95)(0)$ & $(2.24-2.77)(0)$ & $(1.82-2.01)(0)$ \\
\hline$(6.26,3)$ & $(1.47,1.48)(1)$ & $(1.43)$ & $(1.54,1.55)(1)$ & $(2.16-2.28)(1)$ & $(1.61-1.82)(1)$ & $(1.43-1.55)(1)$ \\
\hline
\end{tabular}

The double differential cross section was first computed for all final states, scattering angles and neutrino energies. The total cross section was then calculated by summing up the contribution coming from each nuclear final state and integrating over the scattering angle.

We present in Tables VI and VII our computed total cross sections for neutrino and antineutrino scattering, respectively. The total cross sections increase strongly, as expected, with increasing (anti)neutrino energy. The chosen value of the pairing strength $\left(G_{n}\right.$ or $\left.G_{p}\right)$ has a very small impact on the total cross section, in particular for the neutrino scattering.

It is possible to compare our computed total cross sections for neutrino scattering off ${ }^{208} \mathrm{~Pb}$ with two previous calculations $[24,25]$. The nuclear structure in [24] has been dealt with by the RPA where the single-particle energies were obtained from a Woods-Saxon potential, and for the two-body interaction the zero-range Landau-Migdal force was used. The QRPA used in [25] is based on a self-consistent mean field and for the interaction the Skyrme force SIII was adopted. In our computations we use, as described in Sec. II A, the pnQRPA to construct the participant nuclear wave functions. A selfconsistent mean field and two-body interactions of the Skyrme type were used. This comparison of the different approaches is displayed in Fig 2. In Fig. 2 we display the total cross sections as functions of the neutrino energy. It can be noticed in the lower panel of the figure that our obtained cross sections are smaller than the ones predicted in [24,25] for neutrino energies $E_{v}>30 \mathrm{MeV}$. Our total cross sections are in very good agreement with those of [24] for the $\mathrm{SkM}^{*}$ force and $E_{v}=10-25 \mathrm{MeV}$, and for the SkX force for neutrino energies around $30 \mathrm{MeV}$. The difference between our computed cross sections and those predicted in Refs. [24,25] increases with increasing neutrino energy.

It not easy to track down the reason for the deviations in the three calculations. Reference [24] uses a different type of interaction and possibly a different approach for the final-state
Coulomb effects. For large values of the energy of the incoming neutrino also the energy of the final-state lepton is high, on average, and treatment of these high final-state energies by the bare Fermi function [24] or the MEMA discussed in Sec. II B
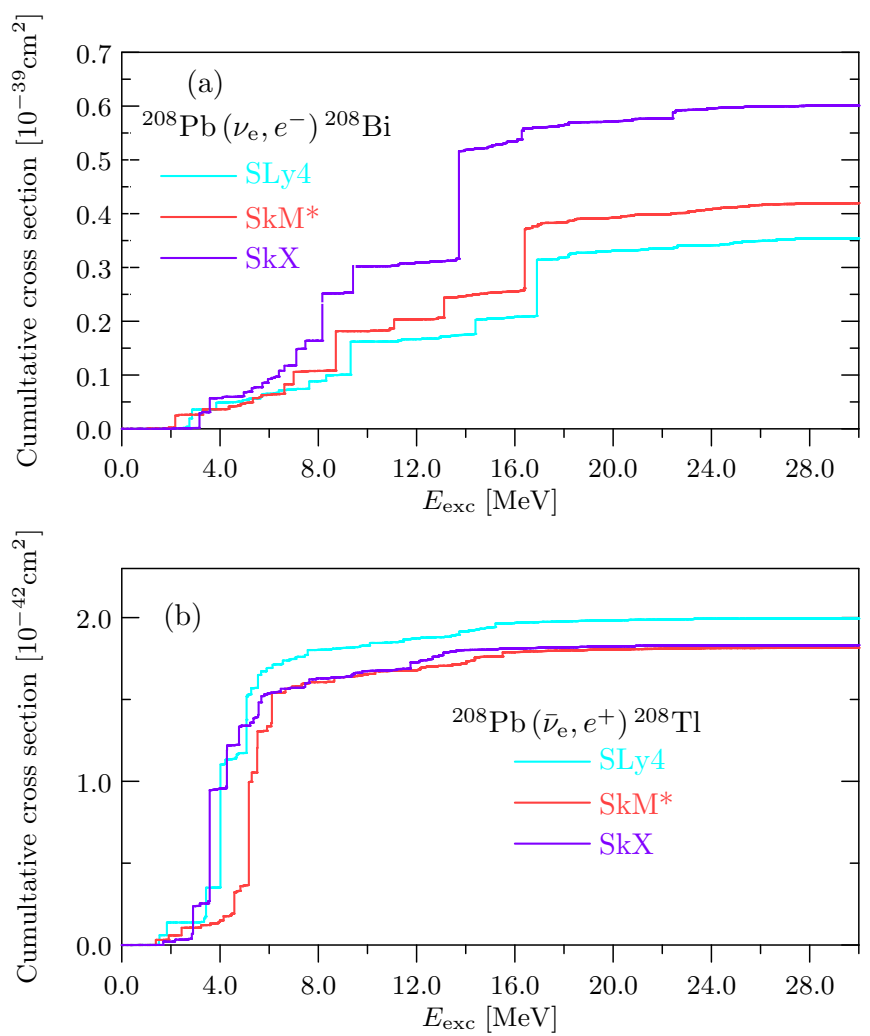

FIG. 4. Cumulative sums of the averaged cross section for the neutrino (a) and antineutrino (b) scattering off ${ }^{208} \mathrm{~Pb}$ computed using the three considerd Skyrme interactions. The (anti)neutrino parameters are $(T, \alpha)=(4,3)$. The pairing strength is $G_{n}$. 

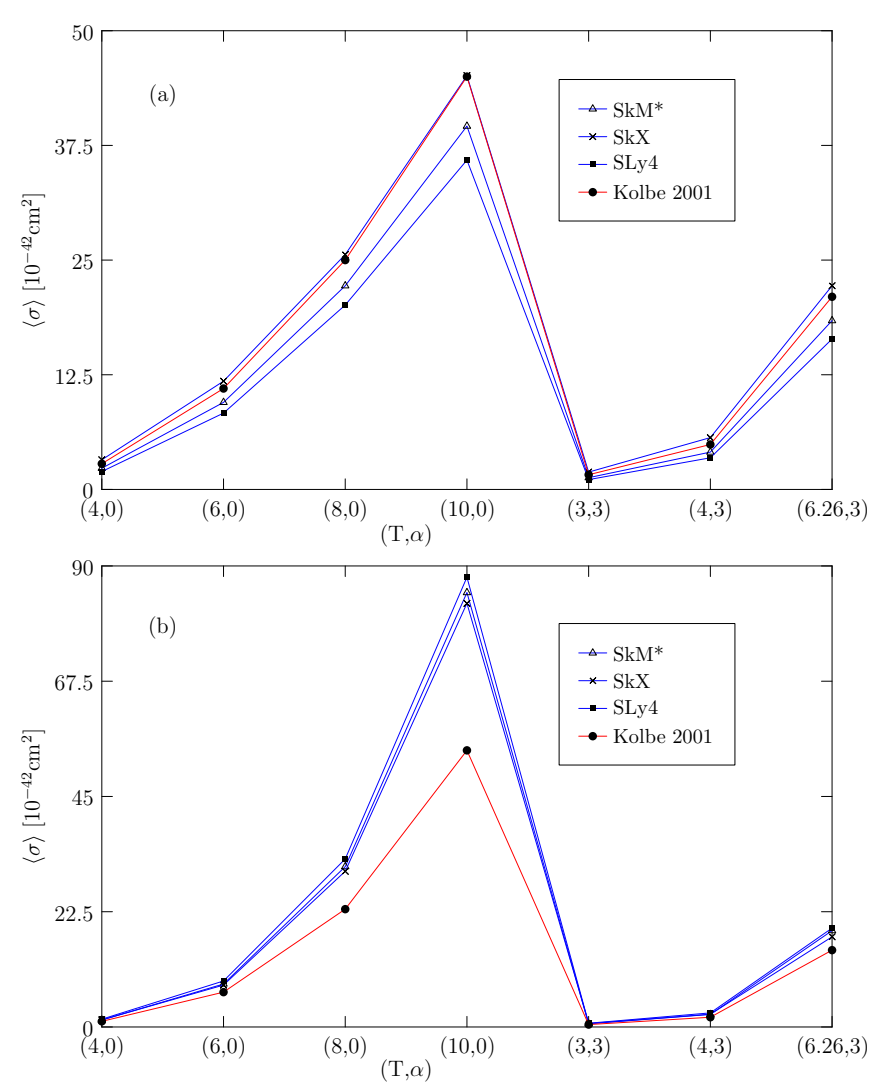

FIG. 5. Comparison between our averaged cross sections and those of Kolbe 2001, i.e., Ref. [24], for the charged-current neutrino (a) and antineutrino (b) scattering off ${ }^{208} \mathrm{~Pb}$.

makes a difference. In the case of Ref. [25] the difference with the present results is harder to explain, although there the SIII Skyrme force was employed instead of those used in the present work. In [25], as also in the present work, the MEMA was used to take into account the final-state Coulomb effects. In particular, the large bump in the interval $20-40 \mathrm{MeV}$ (see Fig. 2) in the calculation of [25] deviates conspicuously from our result and the one of [24]. This deviation is even more mysterious when contrasted with similar Skyrme SIII calculations reported in [44,45], where the obtained cross sections were very close to the results of [24] for all neutrino energies. In Fig. 3 we compare our computed antineutrino cross sections with the one of Ref. [25] for ${ }^{208} \mathrm{~Pb}$. Here the difference between the present results and those of [25] is striking. The reason for this conspicuous difference is unknown.

\section{Averaged cross sections}

The flux-averaged cross section, which is of interest from the experimental point of view, is computed in this work by folding the obtained total cross sections with the appropriate energy profile for the incoming (anti)neutrinos. We have used in the present work the two-parameter Fermi-Dirac distribution which describes usually reasonably the energy spectra of supernova neutrinos. It is given by

$$
F_{\mathrm{FD}}\left(E_{\mathbf{k}}\right)=\frac{1}{F_{2}\left(\alpha_{v}\right) T_{v}} \frac{\left(E_{\mathbf{k}} / T_{\nu}\right)^{2}}{1+\exp \left(E_{\mathbf{k}} / T_{\nu}-\alpha_{\nu}\right)},
$$

where $T_{v}$ is the effective neutrino temperature and $\alpha_{v}$ represents the so-called pinching parameter. The constant $F_{2}\left(\alpha_{v}\right)$ is chosen in such a way that it normalizes the total flux to unity.

The adopted values of the parameters $T_{\nu}$ and $\alpha_{\nu}$, and the resulting averaged cross sections for neutrino and antineutrino scattering off the considered lead isotopes are tabulated in Tables VIII and IX. In the "Full range" column we summarize all the Skyrme results for each nucleus by giving a range of cross sections running from the smallest to the largest predicted cross section. It is clear from the tables that the $\mathrm{SkX}$ interaction predicts the largest averaged neutrino cross sections while the SLy4 predicts the smallest ones. The results presented under column "Full range" are practically independent of the nucleus. For the antineutrino scattering, the relative magnitudes of the averaged cross sections, computed by using the different interactions, depend on both the nucleus and the supernova model $(T, \alpha)$. The largest (smallest) averaged antineutrino cross sections are obtained for ${ }^{204} \mathrm{~Pb}\left({ }^{208} \mathrm{~Pb}\right)$.

An intriguing question concerning the total averaged cross sections is which final states contribute to them the most. The relevant final-state energy intervals can be studied by plotting the cumulative cross sections as functions of the excitation energy of the final nucleus. This has been done in Fig. 4 for the neutrino and antineutrino scattering off ${ }^{208} \mathrm{~Pb}$. The plots have been done for $(T, \alpha)=(4,3)$ and for the pairing strength $G_{n}$.

In Fig. 4(a) one can see that SkX (SLy4) has the largest (smallest) total cross section, as inferred already from Table VIII. The major contribution for each interaction comes from the GTGR in the range 13-17 MeV (see Table I). Beyond the GTGR contribution the total cross section is practically

TABLE X. Contributions of the dominant multipole channels to the averaged cross sections, with parameters $T=2.88$ and $\alpha=3$, for the charged-current neutrino scattering in units of $10^{-42} \mathrm{~cm}^{2}$. The values on the left (right) correspond to pairing strength $G_{n}\left(G_{p}\right)$.

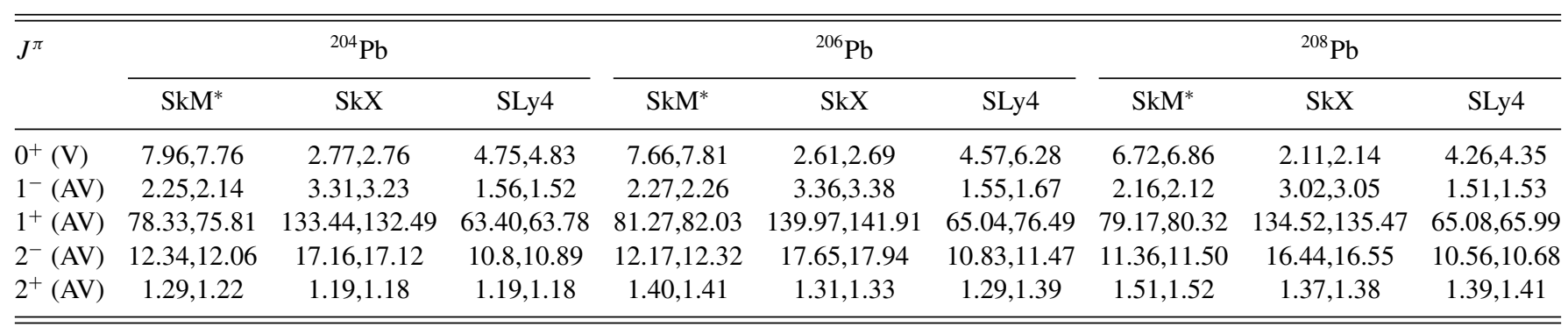


TABLE XI. Same as Table X for the charged-current antineutrino scattering in units of $10^{-43} \mathrm{~cm}^{2}$. The antineutrino temperature here is $T=3.41$ and the pinching parameter is $\alpha=3$.

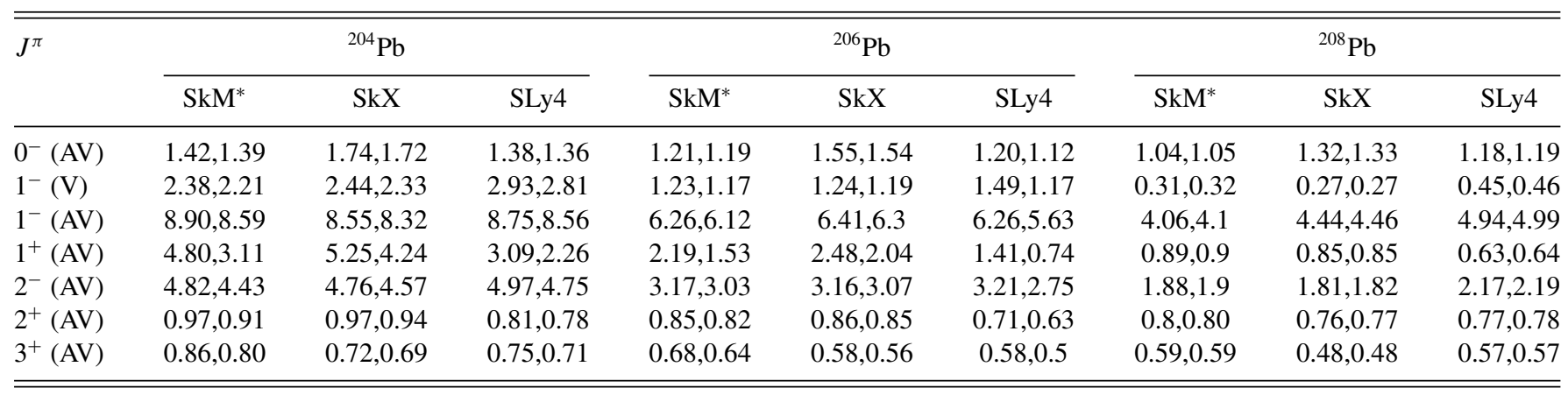

saturated. In all cases a tiny contribution comes from the IAS, showing up as a small step at the location of the IAS. A lot of other notable contributions (mostly GT) are visible within the range 1-14 MeV. Overall, the cumulative sum for neutrino scattering off ${ }^{208} \mathrm{~Pb}$ in panel (a) of Fig. 4 resembles closely the corresponding cumulative $\beta^{-}$strength in Fig 1. Similar results can be found for ${ }^{204} \mathrm{~Pb}$ and ${ }^{206} \mathrm{~Pb}$.

In the case of the antineutrino scattering the cumulative sums for the three interactions and three $\mathrm{Pb}$ nuclei saturate at around 5-9 MeV, much earlier than for the neutrino scattering. In contrast to the neutrino scattering, the relative magnitudes of the cumulative sums for the antineutrino scattering vary from nucleus to nucleus. In particular, for ${ }^{208} \mathrm{~Pb}$ the largest cross section is obtained by the SLy4 interaction instead of the SkX interaction. Also, the relevant contributions to antineutrino scattering off ${ }^{208} \mathrm{~Pb}$ come from much lower energies than in the case of ${ }^{204,206} \mathrm{~Pb}$.

For the sake of comparison with the averaged cross sections in Ref. [24], we have recomputed our averaged cross sections for the neutrino and antineutrino scatterings off ${ }^{208} \mathrm{~Pb}$ with their quenching factor $(0.7)^{2}$. The value $(1 / 1.267)^{2}$ was used before in our computations. The new averaged cross sections are presented in Fig. 5 together with the numbers from Ref. [24]. As can be seen in Fig. 5, quite a good agreement with Ref. [24] has been achieved for neutrino scattering, in particular for the SkX interaction. Our averaged antineutrino cross sections are slightly larger than those in Ref. [24], particularly for $T_{v}=$ 10 , but overall the correspondence is good. The results of Fig. 5(a) reflect directly the situation in Fig. 2(a), where SkX gives larger cross sections than $\mathrm{SkM}^{*}$ and SLy4 (in that order). The order of the folded cross sections of the Skyrme results in Fig. 5(a) is independent of the temperature since for all energies of the incoming neutrino the cross sections are in descending order from $\mathrm{SkX}$ to $\mathrm{SkM}^{*}$ to $\mathrm{SLy} 4$. The relative magnitudes of the SkX and Ref. [24] results in Fig. 5(a) depend on the temperature $T$ : For $T \lesssim 8 \mathrm{MeV}$ the energy profile (17) picks contributions of the cross sections of Fig. 2(a) in the energy range $E_{\mathbf{k}} \lesssim 30 \mathrm{MeV}$ and the SkX cross section is larger than the one of Ref. [24], as seen in Fig. 5(a). For $T \approx 10$ $\mathrm{MeV}$ the cross-section contributions come mainly from the energy range $E_{\mathbf{k}} \lesssim 40 \mathrm{MeV}$ and the two folded results are practically the same since beyond $E_{\mathbf{k}}=30 \mathrm{MeV}$ the cross sections of [24] are larger than the SkX cross sections. From Fig. 5(b) it is then easy to infer that the antineutrino cross sections of [24] lie below those of SkX for neutrino energies $E_{\mathbf{k}} \gtrsim 20 \mathrm{MeV}$.

We have investigated the contributions coming from the leading multipole channels to the averaged cross sections. The results for neutrino and antineutrino reactions are presented in Tables X and XI, respectively. Related to this, we show in Fig. 6 representative plots for the neutrino and antineutrino scatterings off ${ }^{208} \mathrm{~Pb}$. In this Fig. we display the vector, axial-vector, and interference contributions to each multipole $J^{\pi}$. It can be concluded from Table $X$ that the neutrino scattering is dominated by the $1^{+}$multipole of axial-vector
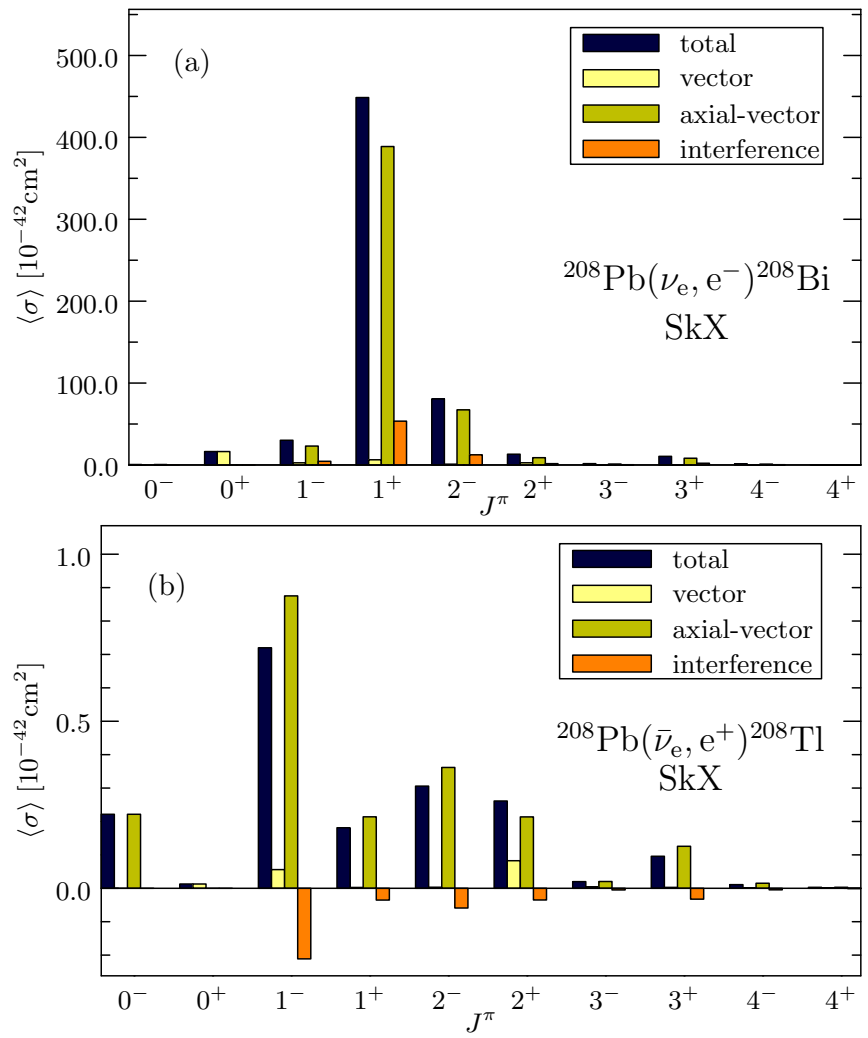

FIG. 6. Contributions of the various multipole channels to the averaged cross sections of the antineutrino (a) and neutrino (b) scattering off ${ }^{208} \mathrm{~Pb}$. The folding parameters in these plots are $(T, \alpha)=(4,3)$ and the pairing strength is $G_{n}$. 
TABLE XII. Dominant transitions for the averaged charged-current (anti)neutrino scattering off ${ }^{208} \mathrm{~Pb}$. The (anti)neutrino parameters are $(T, \alpha)=(4,3)$. The triplet of numbers in the parentheses are first the spin-parity and ordinal $\left(J_{k}^{\pi}\right)$, second the excitation energy in units of MeV, and third the cross section in units of $10^{-41} \mathrm{~cm}^{2}$.

\begin{tabular}{|c|c|c|c|c|}
\hline Scattering & Pairing strength & $\mathrm{SkM}^{*}$ & SkX & SLy4 \\
\hline \multirow[t]{5}{*}{ Neutrino } & $G_{n}$ & $\left(1_{1}^{+}, 2.23,2.18\right)$ & $\left(2_{16}^{-}, 3.18,2.7\right)$ & $\left(1_{1}^{+}, 2.83,1.53\right)$ \\
\hline & & $\left(2_{32}^{-}, 6.63,1.74\right)$ & $\left(1_{2}^{+}, 3.6,2.57\right)$ & $\left(2_{12}^{-}, 2.89,1.65\right)$ \\
\hline & & $\left(1_{23}^{+}, 7.06,2.3\right)$ & $\left(1_{25}^{+}, 7.12,2.99\right)$ & $\left(1_{24}^{+}, 7.66,1.38\right)$ \\
\hline & & $\left(1_{29}^{+}, 8.72,7.31\right)$ & $\left(1_{30}^{+}, 8.19,8.77\right)$ & $\left(1_{29}^{+}, 9.33,6.13\right)$ \\
\hline & & $\begin{array}{c}\left(0_{24}^{+}, 13.12,3.73\right) \\
\left(1_{86}^{+}, 16.43,11.01\right)\end{array}$ & $\begin{array}{c}\left(1_{42}^{+}, 9.42,4.86\right) \\
\left(1_{77}^{+}, 13.74,20.02\right)\end{array}$ & $\begin{array}{c}\left(0_{26}^{+}, 14.42,2.63\right) \\
\left(1_{82}^{+}, 16.9,10.42\right)\end{array}$ \\
\hline Antineutrino & $G_{p}$ & $\begin{array}{l}\left(2_{23}^{-}, 4.52,1.31\right) \\
\left(1_{17}^{-}, 5.11,6.32\right) \\
\left(1_{17}^{+}, 5.26,0.57\right) \\
\left(2_{26}^{-}, 5.46,1.52\right) \\
\left(2_{26}^{+}, 5.46,1.01\right) \\
\left(0_{7}^{-}, 6.05,1.86\right)\end{array}$ & $\begin{array}{c}\left(2_{14}^{-}, 2.9,1.7\right) \\
\left(1_{14}^{-}, 3.55,6.82\right) \\
\left(0_{6}^{-}, 4.25,2.2\right) \\
\left(2_{24}^{-}, 4.77,1.12\right)\end{array}$ & $\begin{array}{l}\left(2_{15}^{-}, 3.39,1.77\right) \\
\left(1_{15}^{-}, 4.01,7.56\right) \\
\left(0_{6}^{-}, 5.02,2.03\right) \\
\left(2_{23}^{-}, 5.02,1.43\right)\end{array}$ \\
\hline
\end{tabular}

type. The antineutrino scattering (see Table XI) is dominated by the spin-dipole $1^{-}$multipole and it is mainly of axial-vector type. The spin-dipole $0^{-}$and $2^{-}$multipoles have notable contributions of axial-vector type. The $1^{+}$multipole of axialvector type contributes notably for the antineutrino scattering off ${ }^{204,206} \mathrm{~Pb}$.

In Table XII we show the normalized averaged differential cross sections and the excitation energy of the dominating final states for neutrino and antineutrino scattering off ${ }^{208} \mathrm{~Pb}$. The normalization was done by dividing the differential cross sections by the total averaged cross section; i.e., the sum of all contributions of the excited states is 1 . The excitation energies in the aforementioned table are measured relative to the ground state of ${ }^{208} \mathrm{Bi}$ for neutrino scattering and ${ }^{208} \mathrm{Tl}$ for antineutrino scattering. However, the excitation energies relative to the ground state of ${ }^{208} \mathrm{~Pb}$ can be computed using Eq. (16). We display in Fig. 7 the prominent final states for the neutrino and antineutrino scattering off the lead isotopes with the SkX interactions. It can be concluded that the supernova neutrino scattering off the stable even-even isotopes is dominated by transition to various $1^{+}$states with excitation energy in the range $0.8-16.9 \mathrm{MeV}$. The most prominent final states for the antineutrino scattering are the low-lying $1^{-}$ states. Significant transitions to $0^{-}$and $2^{-}$states can also be noticed. The transitions to $1^{+}$states are also important for antineutrino scattering off ${ }^{204} \mathrm{~Pb}$. The bulk of the cross sections for antineutrino scattering are coming from several states with excitation energies $0-10 \mathrm{MeV}$.

\section{Effects of the neutrino oscillations}

Only the electron neutrino and electron antineutrino can be detected in the CC supernova-neutrino detection experiments because the large muon and tau rest masses cannot be created in the low-energy supernova-neutrino processes. However, neutrinos undergo flavor conversions when they propagate through the dense matter of the star. Collective neutrino oscillations, caused by neutrino-neutrino interactions, have significant effects on the energy profiles of supernova neutrinos [46]. It is usually assumed that the energy spectra of muon and tau neutrinos are the same. It can then be shown that the threeneutrino mixing problem can be reduced to a two-neutrino problem of the form $v_{x} \rightarrow v_{e}$, where $v_{x}$ is a linear combination of $v_{\mu}$ and $v_{\tau}[47,48]$. Consequently, the energy profile for the electron neutrinos which reach an Earth-bound detector can then by written in the form

$$
F_{v_{e}}\left(E_{\mathbf{k}}\right)=p\left(E_{\mathbf{k}}\right) F_{v_{e}}^{0}\left(E_{\mathbf{k}}\right)+\left[1-p\left(E_{\mathbf{k}}\right)\right] F_{v_{x}}^{0}\left(E_{\mathbf{k}}\right),
$$

where $p\left(E_{\mathbf{k}}\right)$ represents the survival probability of the electron neutrinos and $F_{v_{e}}^{0}\left(E_{\mathbf{k}}\right)\left[F_{v_{x}}^{0}\left(E_{\mathbf{k}}\right)\right]$ is the initial energy profile of the electron neutrinos [nonelectron neutrinos].

For the electron antineutrinos one has an analogous expression

$$
F_{\bar{\nu}_{e}}\left(E_{\mathbf{k}}\right)=\bar{p}\left(E_{\mathbf{k}}\right) F_{\bar{\nu}_{e}}^{0}\left(E_{\mathbf{k}}\right)+\left[1-\bar{p}\left(E_{\mathbf{k}}\right)\right] F_{\bar{\nu}_{x}}^{0}\left(E_{\mathbf{k}}\right),
$$

with corresponding quantities of (18) where neutrino is replaced by antineutrino. 

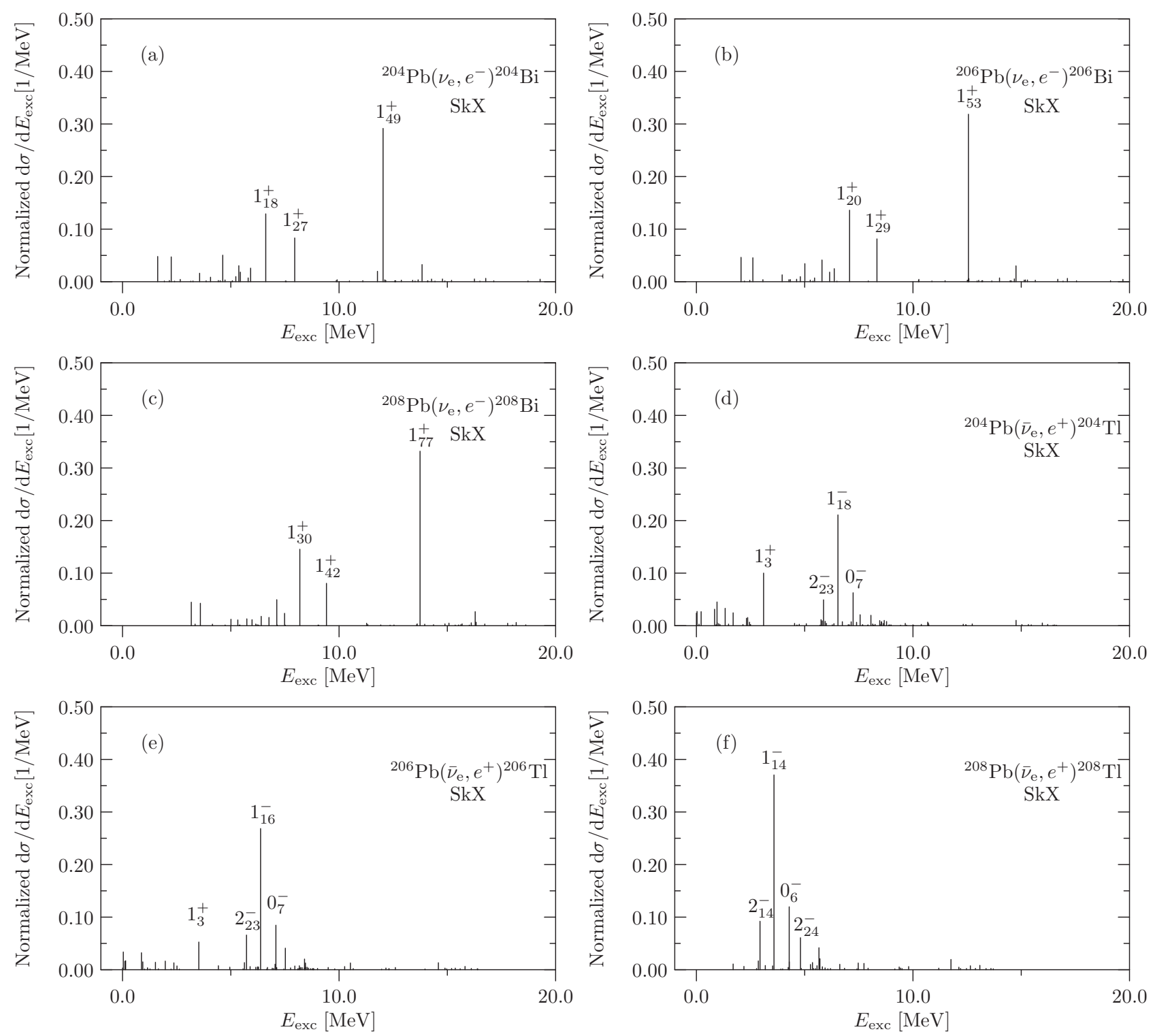

FIG. 7. Contributions from the final nuclear states to the normalized averaged differential cross sections for the neutrino [panels (a)-(c)] and antineutrino [panels (d)-(f)] scattering off lead isotopes for the SkX interaction. The (anti)neutrino parameters are $T=4$ and $\alpha=3$, and the pairing strength is $G_{n}$.

We adopted the prescription of $[49,50]$ for the survival probabilities $p\left(E_{\mathbf{k}}\right)$ and $\bar{p}\left(E_{\mathbf{k}}\right)$ of the electron neutrinos and electron antineutrinos. In the case of the normal mass hierarchy we have

$$
p\left(E_{\mathbf{k}}\right)=0,
$$

and

$$
\bar{p}\left(E_{\mathbf{k}}\right)= \begin{cases}\cos \theta_{12}, & E_{\mathbf{k}}<\bar{E}_{s}, \\ 0, & E_{\mathbf{k}}>\bar{E}_{s},\end{cases}
$$

where $\bar{E}_{s}=18.0 \mathrm{MeV}$ [50]. For the inverted mass hierarchy we adopt the survival probabilities

$$
p\left(E_{\mathbf{k}}\right)= \begin{cases}\sin ^{2} \theta_{12}, & E_{\mathbf{k}}<E_{s} \\ 0, & E_{\mathbf{k}}>E_{s}\end{cases}
$$

and

$$
\bar{p}\left(E_{\mathbf{k}}\right)=\cos ^{2} \theta_{12},
$$

for the electron neutrinos and electron antineutrinos, respectively. Here we choose the value $E_{s}=7 \mathrm{MeV}$ [47]. In the present computations we employ for the mixing angle the values of [51], i.e., $\sin ^{2} \theta_{12}=0.306$ for the normal hierarchy and $\sin ^{2} \theta_{12}=0.312$ for the inverted hierarchy.

For the present study of neutrino-oscillation effects we have adopted the neutrino and antineutrino parameters from [52]. The values of these parameters are shown in Table XIII. We present in Table XIV the resulting averaged cross sections for the nonoscillating and oscillating electron (anti)neutrinos. In this table, the labels $v_{e}$ and $\bar{v}_{e}$ stand for the nonoscillating electron neutrino and electron antineutrino, respectively. The results for the oscillating electron neutrino (electron 
TABLE XIII. Values of the parameters $\alpha, T$ and the average neutrino energies for three different supernova scenarios adopted from [52]. In this table $x$ denotes the nonelectron flavors, i.e., $x=\mu, \tau$.

\begin{tabular}{lllll}
\hline \hline & $\left(\alpha_{v_{e}}, T_{v_{e}},\left\langle E_{v_{e}}\right\rangle\right)$ & $\left(\alpha_{\bar{v}_{e}}, T_{\bar{v}_{e}},\left\langle E_{\bar{v}_{e}}\right\rangle\right)$ & $\left(\alpha_{v_{\mathrm{x}}}, T_{v_{\mathrm{x}}},\left\langle E_{v_{\mathrm{x}}}\right\rangle\right)$ & $\left(\alpha_{\bar{v}_{\mathrm{x}}}, T_{\bar{v}_{\mathrm{x}}},\left\langle E_{\bar{v}_{\mathrm{x}}}\right\rangle\right)$ \\
\hline (I) & $(3.0,2.88,11.5)$ & $(3.0,3.41,13.6)$ & $(3.0,4.08,16.3)$ & $(3.0,4.08,16.3)$ \\
(II) & $(0.0,3.65,11.5)$ & $(0.0,4.32,13.6)$ & $(0.0,5.17,16.3)$ & $(0.0,5.17,16.3)$ \\
(III) & $(3.0,2.88,11.5)$ & $(3.0,3.41,13.6)$ & $(0.0,5.17,16.3)$ & $(0.0,5.17,16.3)$ \\
\hline \hline
\end{tabular}

antineutrino) are denoted by $v_{\mathrm{ex}}^{\mathrm{NH}}\left(\bar{\nu}_{\mathrm{ex}}^{\mathrm{NH}}\right)$ for the normal mass hierarchy and by $v_{\mathrm{ex}}^{\mathrm{IH}}\left(\bar{v}_{\mathrm{ex}}^{\mathrm{IH}}\right)$ for the inverted mass hierarchy. Furthermore, in the rows labeled "Full" we summarize the results of the considered Skyrme interactions by giving ranges which run from the smallest to the largest predicted value. It is seen in Table XIV that the flux-averaged cross sections are enhanced significantly when the flavor transformations are included. The effect of the chosen pairing strength on the averaged cross sections is very weak. It can also be concluded from Table XIV that the averaged cross sections for the oscillating electron neutrino are almost the same for the normal and inverted mass hierarchies. For the electron antineutrino,

TABLE XIV. Averaged cross sections for the charged-current (anti)neutrino-nucleus scatterings in units of $10^{-41} \mathrm{~cm}^{2}$ as calculated for the sets of neutrino parameters quoted in Table XIII. Here the columns $v_{e}$ and $\bar{v}_{e}$ are computed without taking into account the neutrino oscillations, while $v_{\mathrm{ex}}^{\mathrm{NH}}$ (normal hierarchy) and $v_{\mathrm{ex}}^{\mathrm{IH}}$ (inverted hierarchy) correspond to results with the oscillations included. The values in the rows "Full" run from the smallest to the largest value predicted by the three considered Skyrme interactions. The values on the left (right) correspond to the pairing strength $G_{n}\left(G_{p}\right)$

\begin{tabular}{|c|c|c|c|c|c|c|c|c|}
\hline Nucleus & Model & Int. & $v_{e}$ & $v_{\mathrm{ex}}^{\mathrm{NH}}$ & $v_{\mathrm{ex}}^{\mathrm{IH}}$ & $\bar{v}_{e}$ & $\bar{v}_{\mathrm{ex}}^{\mathrm{NH}}$ & $\bar{v}_{\mathrm{ex}}^{\mathrm{IH}}$ \\
\hline \multirow[t]{10}{*}{${ }^{204} \mathrm{~Pb}$} & \multirow[t]{3}{*}{ (I) } & $\mathrm{SkM}^{*}$ & $12.18,11.78$ & $45.60,44.52$ & $45.44,44.40$ & $0.214,0.188$ & $0.42,0.380$ & $0.278,0.248$ \\
\hline & & $\mathrm{SkX}$ & $18.63,18.49$ & $63.15,62.79$ & $62.40,62.00$ & $0.222,0.206$ & $0.427,0.402$ & $0.285,0.267$ \\
\hline & & Full & $9.74-18.63$ & $38.00-63.15$ & $38.09-62.40$ & $0.188-0.222$ & $0.380-0.427$ & $0.248-0.285$ \\
\hline & \multirow[t]{3}{*}{ (II) } & $\mathrm{SkM}^{*}$ & $17.00,16.52$ & $56.83,55.70$ & $57.54,56.37$ & $0.301,0.272$ & $0.604,0.559$ & $0.395,0.361$ \\
\hline & & SkX & $25.36,25.20$ & $74.55,74.16$ & $74.98,74.60$ & $0.305,0.287$ & $0.605,0.576$ & $0.398,0.377$ \\
\hline & & Full & $13.88-25.36$ & $49.11-74.55$ & $49.72-74.98$ & $0.272-0.305$ & $0.559-0.605$ & $0.361-0.398$ \\
\hline & \multirow[t]{4}{*}{ (III) } & $\mathrm{SkM}^{*}$ & $12.18,11.78$ & $56.83,55.70$ & $57.54,56.37$ & $0.214,0.188$ & $0.612,0.564$ & $0.335,0.303$ \\
\hline & & $\mathrm{SkX}$ & $18.63,18.49$ & $74.55,74.16$ & $74.98,74.60$ & $0.222,0.206$ & $0.613,0.583$ & $0.341,0.321$ \\
\hline & & SLy4 & $9.74,9.80$ & $49.14,49.11$ & $49.72,49.72$ & $0.203,0.189$ & $0.593,0.565$ & $0.322,0.305$ \\
\hline & & Full & $9.74-18.63$ & $49.11-74.55$ & $49.72-74.98$ & $0.188-0.222$ & $0.564-0.613$ & $0.303-0.341$ \\
\hline \multirow[t]{8}{*}{${ }^{206} \mathrm{~Pb}$} & (I) & $\mathrm{SkM}^{*}$ & $12.50,12.62$ & $46.59,46.85$ & $46.44,46.69$ & $0.137,0.127$ & $0.292,0.275$ & $0.186,0.173$ \\
\hline & \multirow{3}{*}{ (II) } & $\mathrm{SkX}$ & $26.49,26.82$ & $77.25,77.87$ & $77.76,78.39$ & $0.215,0.206$ & $0.452,0.438$ & $0.289,0.278$ \\
\hline & & SLy4 & $14.12,15.81$ & $50.00,52.90$ & $50.65,53.71$ & $0.202,0.175$ & $0.435,0.385$ & $0.274,0.240$ \\
\hline & & Full & $14.12-26.82$ & $50.00-77.87$ & $50.65-78.39$ & $0.175-0.215$ & $0.385-0.452$ & $0.240-0.289$ \\
\hline & \multirow[t]{4}{*}{ (III) } & $\mathrm{SkM}^{*}$ & $12.50,12.62$ & $57.93,58.08$ & $58.70,58.88$ & $0.137,0.127$ & $0.452,0.431$ & $0.234,0.221$ \\
\hline & & $\mathrm{SkX}$ & $19.43,19.70$ & $77.25,77.87$ & $77.76,78.39$ & $0.146,0.139$ & $0.456,0.442$ & $0.241,0.232$ \\
\hline & & SLy4 & $9.94,11.55$ & $50.00,52.90$ & $50.65,53.71$ & $0.132,0.111$ & $0.438,0.387$ & $0.226,0.196$ \\
\hline & & Full & 9.94-19.70 & $50.00-77.87$ & $50.65-78.39$ & $0.111-0.146$ & $0.387-0.456$ & $0.196-0.241$ \\
\hline \multirow[t]{4}{*}{${ }^{208} \mathrm{~Pb}$} & \multirow[t]{4}{*}{ (I) } & $\mathrm{SkM}^{*}$ & $12.07,12.25$ & $45.64,46.13$ & $45.55,46.01$ & $0.084,0.085$ & $0.199,0.201$ & $0.120,0.121$ \\
\hline & & SkX & $18.62,18.75$ & $64.34,64.65$ & $63.49,63.79$ & $0.088,0.088$ & $0.200,0.201$ & $0.123,0.124$ \\
\hline & & SLy4 & $9.89,10.02$ & $38.67,39.07$ & $38.82,39.18$ & $0.095,0.096$ & $0.218,0.220$ & $0.134,0.135$ \\
\hline & & Full & $9.89-18.75$ & $38.67-64.65$ & $38.82-63.79$ & $0.084-0.096$ & $0.199-0.220$ & $0.120-0.135$ \\
\hline
\end{tabular}




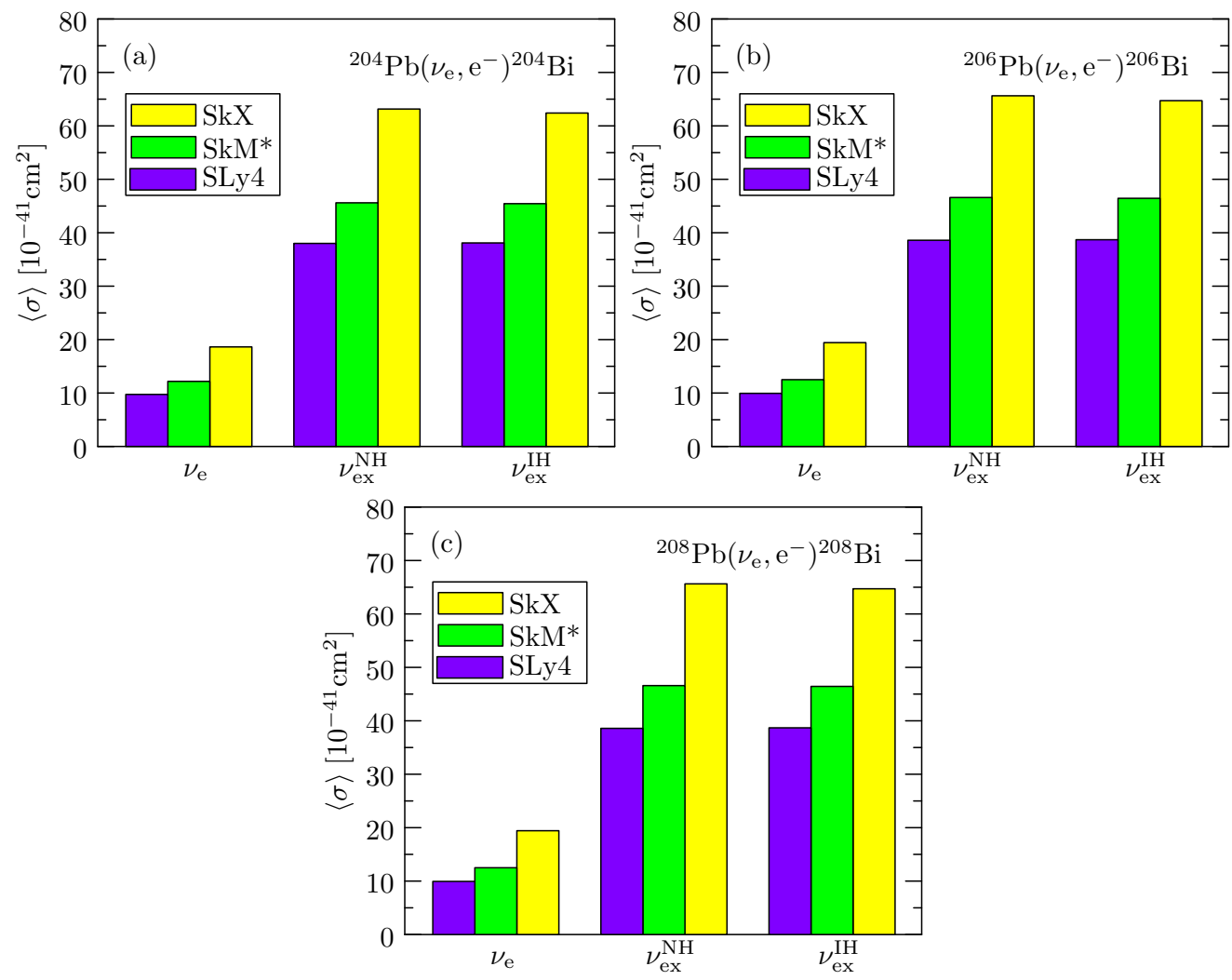

FIG. 8. Computed averaged neutrino-scattering cross sections for the different lead isotopes [panels (a)-(c)] without neutrino oscillations $\left(v_{e}\right)$ and with neutrino oscillations for the normal hierarchy $\left(v_{\mathrm{ex}}^{\mathrm{NH}}\right)$ and inverted hierarchy $\left(v_{\mathrm{ex}}^{\mathrm{IH}}\right)$. Results corresponding to the supernova model (I) of Table XIII for the three adopted Skyrme interactions are shown by vertical bars..

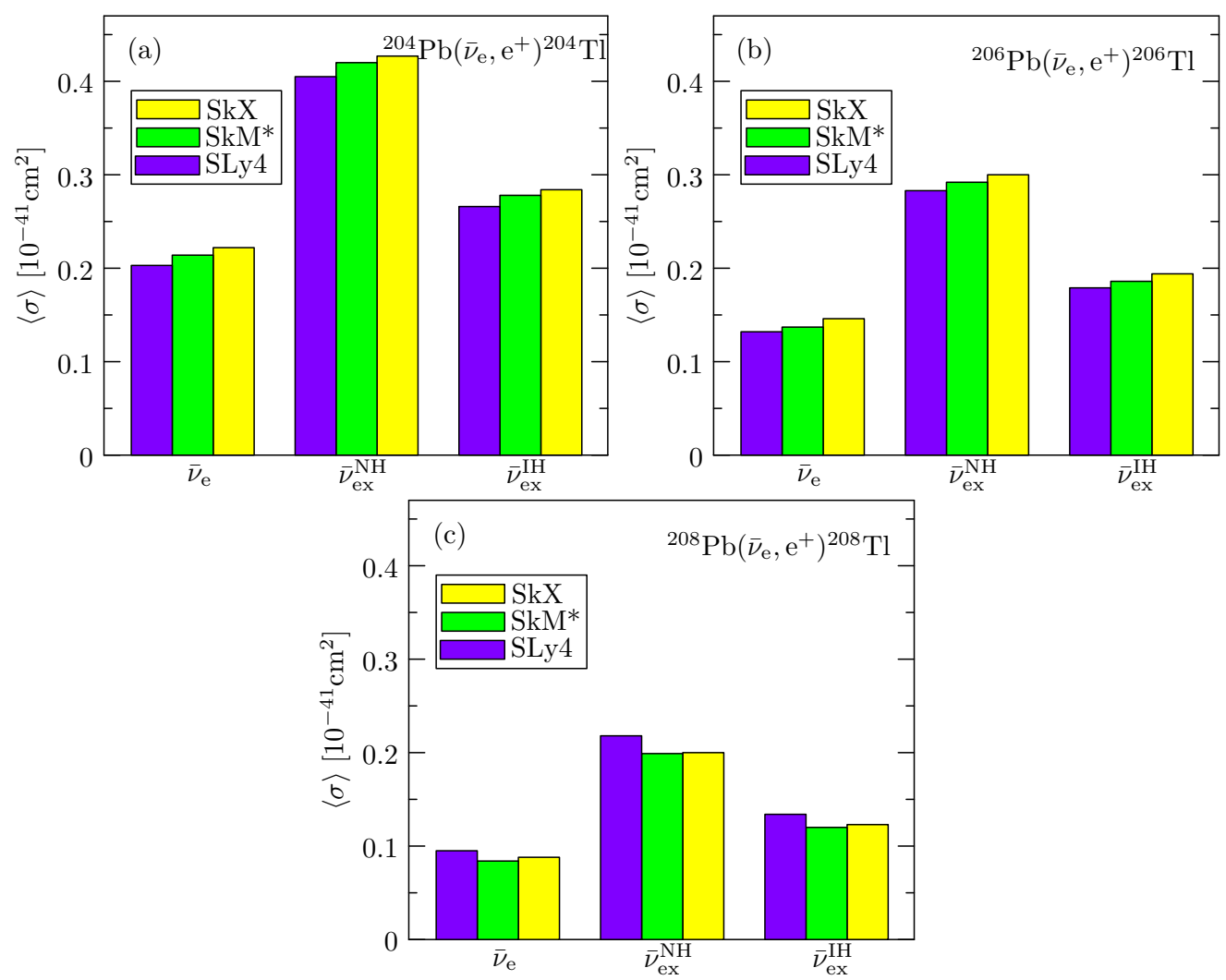

FIG. 9. The same as Fig. 8 for the computed averaged antineutrino-scattering cross sections. 
the results for the normal mass hierarchy are larger than those for the inverted mass hierarchy. These effects of the oscillations on the averaged cross sections are visible in Figs. 8 and 9. In these figures we display the computed averaged cross sections for the supernova model (I) of Table XIII for the discussed lead isotopes. Similar pattern can be seen for the other considered flavor-transformation scenarios (II) and (III). It is clear from Fig. 8 that the neutrino scattering results for the normal hierarchy do not deviate from the results for the inverted hierarchy. Furthermore, the predicted cross sections do not vary notably from one lead isotope to the other. The SkX force gives the largest average cross section for the neutrino-nucleus scattering and the SLy4 gives the smallest. In Fig. 9 the different effects of the two hierarchies on the antineutrino oscillation show up clearly. The averaged cross section for the antineutrino is $A$ dependent and decreases with increasing mass number. It is also evident that the averaged cross sections for the antineutrino-nucleus scattering predicted by the three Skyrme calculations are very close to each other. The most interesting feature is the notable difference between the results obtained for the normal and inverted mass hierarchies. This difference is characteristic for all considered lead isotopes.

\section{CONCLUSIONS}

In this work we have performed calculations for the charged-current (anti)neutrino scattering off the stable eveneven lead isotopes ${ }^{204,206,208} \mathrm{~Pb}$. The cross sections have been calculated for neutrino energies that are appropriate for supernova neutrinos. We have estimated the nuclear responses of the studied lead isotopes by folding the cross sections with a two-parameter Fermi-Dirac distribution describing the energy profile of the incoming (anti)neutrinos. We have compared our obtained total and averaged cross sections with two other calculations in the case of ${ }^{208} \mathrm{~Pb}$. We have also estimated the effect of the flavor conversions of (anti)neutrinos in an exploding supernova on the flux-averaged cross sections.

The nuclear wave functions have been computed within the pnQRPA framework. Our computations were based on the Skyrme interaction variants $\mathrm{SkM}^{*}, \mathrm{SkX}$, and SLy4 as two-body interactions. We have compared our computed $\beta^{-}$GamowTeller strength distribution and the position of the isobaric analog state with the available experimental data for ${ }^{208} \mathrm{~Pb}$.

Our computed results show that the dominant final states for the supernova-neutrino scattering are of the multipole $1^{+}$. The $1^{-}$final states play a prominent role for the antineutrino scattering. The $2^{-}, 0^{-}$, and $1^{+}$channels are also important for the antineutrino scattering.

For the considered scenarios for the (anti)neutrino-flavour transformations we have found that the (anti)neutrino oscillations enhance the (anti)neutrino flux-averaged cross sections. Our results indicate that averaged cross sections for the antineutrinos are quite different for the normal and inverted mass hierarchy. This opens the intriguing possibility to use the future (megaton) neutrino detectors to determine the mass hierarchy (normal or inverted) of the neutrinos.

\section{ACKNOWLEDGMENTS}

This work was supported by the Academy of Finland under the Finnish Center of Excellence Program 2012-2017 (Nuclear and Accelerator Based Program at JYFL).
[1] J. Maalampi and J. Suhonen, Adv. High Energy Phys. 2013, 505874 (2013).

[2] A. B. Balantekin and G. M. Fuller, J. Phys. G: Nucl. Part. Phys. 29, 2513 (2003).

[3] K. Scholberg, Annu. Rev. Nucl. Part. Sci. 62, 81 (2012).

[4] K. Langanke and E. Kolbe, At. Data Nucl. Data Tables 79, 293 (2001).

[5] K. Langanke and G. Martínez-Pinedo, Rev. Mod. Phys. 75, 819 (2003).

[6] H. Ejiri, Phys. Rep. 338, 265 (2000).

[7] K. Langanke, Acta Phys. Pol. B 39, 265 (2008).

[8] Helium and Lead Observatory, http://www.snolab.ca/halo/

[9] K. Zuber, Nucl. Part. Phys. Proc. 265-266, 233 (2015).

[10] J. Suhonen and O. Civitarese, Phys. Rep. 300, 123 (1998).

[11] F. T. Avignone III, S. R. Elliott, and J. Engel, Rev. Mod. Phys. 80, 481 (2008).

[12] C. Volpe, J. Phys. G: Nucl. Part. Phys 31, 903 (2005).

[13] E. Ydrefors, K. G. Balasi, J. Suhonen, and T. S. Kosmas, Neutrinos: Properties, Reactions, Sources and Detection (Nova Science Publishers, Hauppauge, NY, 2011).

[14] E. Ydrefors, K. G. Balasi, T. S. Kosmas, and J. Suhonen, Nucl. Phys. A 896, 1 (2012).

[15] E. Ydrefors and J. Suhonen, Adv. High Energy Phys. 2012, 373946 (2012).

[16] E. Ydrefors and J. Suhonen, Phys. Rev. C 87, 034314 (2013).
[17] W. Almosly, B. G. Carlsson, J. Dobaczewski, J. Suhonen, J. Toivanen, P. Vesely, and E. Ydrefors, Phys. Rev. C 89, 024308 (2014).

[18] W. Almosly, E. Ydrefors, and J. Suhonen, J. Phys. G: Nucl. Part. Phys. 42, 025106 (2015).

[19] W. Almosly, E. Ydrefors, and J. Suhonen, J. Phys. G: Nucl. Part. Phys. 42, 095106 (2015).

[20] E. Ydrefors, J. Suhonen, and Y. M. Zhao, Phys. Rev. C. 91, 014307 (2015).

[21] B. G. Carlsson, J. Dobaczewski, J. Toivanen, and P. Vesely, Comput. Phys. Commun. 181, 1641 (2010).

[22] M. Dutra, O. Lourenco, J. S. Sá Martins, A. Delfino, J. R. Stone, and P. D. Stevenson, Phys. Rev. C 85, 035201 (2012).

[23] J. S. O'Connell, T. W. Donnelly, and J. D. Walecka, Phys. Rev. C 6, 719 (1972).

[24] E. Kolbe and K. Langanke, Phys. Rev. C 63, 025802 (2001).

[25] R. Lazauskas and C. Volpe, Nucl. Phys. A 792, 219 (2007).

[26] B. Brown, Phys. Rev. C 58, 220 (1998).

[27] H. Krivine, J. Treiner, and O. Bohigas, Nucl. Phys. A 336, 155 (1980).

[28] J. Bartel, P. Quentin, M. Brack, C. Guet, and H. B. Håkansson, Nucl. Phys. A 386, 79 (1982).

[29] E. Chabanat, P. Bonche, P. Haensel, J. Meyer, and R. Schaeffer, Nucl. Phys. A 635, 231 (1998). 
[30] P. Veselý, J. Toivanen, B. G. Carlsson, J. Dobaczewski, N. Michel, and A. Pastore, Phys. Rev. C 86, 024303 (2012).

[31] B. G. Carlsson and J. Toivanen, Phys. Rev. C 89, 054324 (2014).

[32] J. D. Walecka, Theoretical Nuclear and Subnuclear Physics (Imperial College Press, London, 2004).

[33] J. Suhonen, From Nucleons to Nucleus: Concepts of Microscopic Nuclear Theory (Springer, Berlin, 2007).

[34] A. Bohr and B. R. Mottelson, Phys. Lett. B 100, 10 (1981).

[35] B. H. Wildenthal, M. S. Curtin, and B. A. Brown, Phys. Rev. C 28, 1343 (1983).

[36] G. Martínez-Pinedo, A. Poves, E. Caurier, and A. P. Zuker, Phys. Rev. C 53, R2602 (1996).

[37] J. Engel, Phys. Rev. C 57, 2004 (1998).

[38] B. G. Carlsson, J. Toivanen, and A. Pastore, Phys. Rev. C 86, 014307 (2012).

[39] B. S. Flanders, R. Madey, B. D. Anderson, A. R. Baldwin, J. W. Watson, C. C. Foster, H. V. Klapdor, and K. Grotz, Phys. Rev. C 40, 1985 (1989).
[40] National Nuclear Data Center, http://www.nndc.bnl.gov

[41] K. Yoshida, Prog. Theor. Exp. Phys. (2013) 113D02.

[42] Li-Gang Cao, X. Roca-Maza, G. Coló, and H. Sagawa, Phys. Rev. C 92, 034308 (2015).

[43] N. Auerbach and A. Klein, Phys. Rev. C 30, 1032 (1984).

[44] C. Volpe, N. Auerbach, G. Colò, and N. Van Giai, Phys. Rev. C 65, 044603 (2002).

[45] J. Engel, G. C. McLaughlin, and C. Volpe, Phys. Rev. D 67, 013005 (2003).

[46] B. Dasgupta, A. Dighe, G. G. Raffelt, and A. Y. Smirnov, Phys. Rev. Lett. 103, 051105 (2009).

[47] J. Gava and C. Volpe, Phys. Rev. D 78, 083007 (2008).

[48] A. B. Balantekin and G. M. Fuller, Phys. Lett. B 471, 195 (1999).

[49] G. G. Raffelt, Prog. Part. Nucl. Phys. 64, 393 (2010).

[50] G. Martínez-Pinedo, B. Ziebarth, T. Fischer, and K. Langanke, Eur. Phys. J. A 47, 98 (2011).

[51] K. Nakumura et al., J. Phys. G: Nucl. Part. Phys. 37, 075021 (2010).

[52] M. T. Keil and G. G. Raffelt, Astrophys. J. 590, 971 (2003). 